\title{
Some generalized difference double sequence spaces defined by a sequence of Orlicz-functions
}

\author{
Kuldip Raj and Sunil K. Sharma \\ School of Mathematics, \\ Shri Mata Vaishno Devi University, \\ Katra-182320, JEKK, India \\ email: kuldeepraj68@rediffmail.com, sunilksharma42@yahoo.co.in
}

\begin{abstract}
In the present paper we introduce some generalized difference double sequence spaces defined by a sequence of Orlicz-functions. We study some topological properties and some inclusion relations between these spaces. We also make an effort to study these properties over n-normed spaces.
\end{abstract}

\section{RESUMEN}

En este artículo introducimos algunos espacios de sucesiones doble-diferencia generalizadas definidas por una sucesión de funciones de Orlicz. Estudiamos algunas propiedades topológicas y algunas relaciones de inclusión entre estos espacios. Además, hacemos un esfuerzo para estudiar estas propiedades en espacios n-normados.

Keywords and Phrases: P-convergent, Orlicz function, sequence spaces, paranorm space, nnormed space

2010 AMS Mathematics Subject Classification: Primary 42B15; Secondary 40C05 


\section{Introduction and Preliminaries}

The initial works on double sequences is found in Bromwich [4]. Later on, it was studied by Hardy [6], Moricz [17], Moricz and Rhoades [18], Tripathy ([33], [34]), Basarir and Sonalcan [2] and many others. Hardy[6] introduced the notion of regular convergence for double sequences. Quite recently, Zeltser [36] in her Ph.D thesis has essentially studied both the theory of topological double sequence spaces and the theory of summability of double sequences. Mursaleen and Edely [21] have recently introduced the statistical convergence and Cauchy convergence for double sequences and given the relation between statistical convergent and strongly Cesaro summable double sequences. Nextly, Mursaleen [19] and Mursaleen and Edely [22] have defined the almost strong regularity of matrices for double sequences and applied these matrices to establish a core theorem and introduced the $M$-core for double sequences and determined those four dimensional matrices transforming every bounded double sequences $x=\left(x_{m n}\right)$ into one whose core is a subset of the M-core of $x$. More recently, Altay and Basar [1] have defined the spaces $\mathcal{B S}, \mathcal{B S}(\mathrm{t}), \mathcal{C} \mathcal{S}_{\mathrm{p}}, \mathcal{C} \mathcal{S}_{\mathrm{bp}}, \mathcal{C} \mathcal{S}_{\mathrm{r}}$ and $\mathcal{B V}$ of double sequences consisting of all double series whose sequence of partial sums are in the spaces $\mathcal{M}_{\mathfrak{u}}$, $\mathcal{M}_{\mathfrak{u}}(\mathrm{t}), \mathcal{C}_{\mathrm{p}}, \mathcal{C}_{\mathrm{bp}}, \mathcal{C}_{\mathrm{r}}$ and $\mathcal{L}_{\mathfrak{u}}$, respectively and also examined some properties of these sequence spaces and determined the $\alpha$-duals of the spaces $\mathcal{B S}, \mathcal{B V}, \mathcal{C S}_{\mathrm{bp}}$ and the $\beta(v)$-duals of the spaces $\mathcal{C} \mathcal{S}_{\mathrm{bp}}$ and $\mathcal{C} \mathcal{S}_{\mathrm{r}}$ of double series. Now, recently Basar and Sever [3] have introduced the Banach space $\mathcal{L}_{\mathrm{q}}$ of double sequences corresponding to the well known space $\ell_{\mathrm{q}}$ of single sequences and examined some properties of the space $\mathcal{L}_{\mathrm{q}}$. Let $w^{2}$ denote the set of all double sequences of complex numbers. By the convergence of a double sequence we mean the convergence of the Pringsheim sense i.e. a double sequence $x=\left(x_{k l}\right)$ has Pringsheim limit L (denoted by $\mathrm{P}-\lim x=\mathrm{L}$ ) provided that given $\epsilon>0$ there exists $n \in N$ such that $\left|x_{k l}-L\right|<\epsilon$ whenever $k, l>n$ see [26]. We shall write more briefly as P-convergent. We shall denote the space of all P-convergent sequences by $c^{2}$. The double sequence $x=\left(x_{k l}\right)$ is bounded if there exists a positive number $M$ such that $\left|x_{k l}\right|<M$ for all $k$ and $l$. Let $l_{\infty}^{2}$ the space of all bounded double sequence such that $\left\|x_{k l}\right\|_{\infty, 2}=\sup _{k l}\left|x_{k l}\right|<\infty$. For more details about double sequence spaces see ([30], [31],[32]) and references therein.

The notion of difference sequence spaces was introduced by Kızmaz [13], who studied the difference sequence spaces $l_{\infty}(\Delta), c(\Delta)$ and $c_{0}(\Delta)$. The notion was further generalized by Et. and Çolak [5] by introducing the spaces $l_{\infty}\left(\Delta^{n}\right), c\left(\Delta^{n}\right)$ and $c_{0}\left(\Delta^{n}\right)$. Let $w$ be the space of all complex or real sequences $x=\left(x_{k}\right)$ and let $m, s$ be non-negative integers, then for $Z=l_{\infty}, c, c_{0}$ we have sequence spaces

$$
\mathrm{Z}\left(\Delta_{\mathrm{s}}^{\mathrm{m}}\right)=\left\{\mathrm{x}=\left(\mathrm{x}_{\mathrm{k}}\right) \in \mathrm{w}:\left(\Delta_{\mathrm{s}}^{\mathrm{m}} \mathrm{x}_{\mathrm{k}}\right) \in \mathrm{Z}\right\},
$$

where $\Delta_{s}^{m} x=\left(\Delta_{s}^{m} x_{k}\right)=\left(\Delta_{s}^{m-1} x_{k}-\Delta_{s}^{m-1} x_{k+1}\right)$ and $\Delta_{s}^{0} x_{k}=x_{k}$ for all $k \in \mathbb{N}$, which is equivalent to the following binomial representation

$$
\Delta_{\mathrm{s}}^{\mathrm{m}} \mathrm{x}_{\mathrm{k}}=\sum_{v=0}^{\mathrm{m}}(-1)^{v}\left(\begin{array}{c}
\mathrm{m} \\
v
\end{array}\right) \mathrm{x}_{\mathrm{k}+\mathrm{s} v} .
$$

Taking $s=1$, we get the spaces which were studied by Et and Çolak [5]. Taking $m=s=1$, we get the spaces which were introduced and studied by Kızmaz [13]. 
An Orlicz function $M:[0, \infty) \rightarrow[0, \infty)$ is a continuous, non-decreasing and convex function such that $M(0)=0, M(x)>0$ for $x>0$ and $M(x) \longrightarrow \infty$ as $x \longrightarrow \infty$. Lindenstrauss and Tzafriri [14] used the idea of Orlicz function to define the following sequence space:

$$
l_{M}=\left\{x \in w: \sum_{k=1}^{\infty} M\left(\frac{\left|x_{k}\right|}{\rho}\right)<\infty\right\}
$$

which is called as an Orlicz sequence space. Also $l_{M}$ is a Banach space with the norm

$$
\|x\|=\inf \left\{\rho>0: \sum_{k=1}^{\infty} M\left(\frac{\left|x_{k}\right|}{\rho}\right) \leq 1\right\} .
$$

Also, it was shown in [14] that every Orlicz sequence space $l_{M}$ contains a subspace isomorphic to $l_{p}(p \geq 1)$. The $\Delta_{2}-$ condition is equivalent to $M(L x) \leq L M(x)$, for all $L$ with $0<L<1$. An Orlicz function $M$ can always be represented in the following integral form

$$
M(x)=\int_{0}^{x} \eta(t) d t
$$

where $\eta$ is known as the kernel of $M$, is right differentiable for $t \geq 0, \eta(0)=0, \eta(t)>0, \eta$ is non-decreasing and $\eta(t) \rightarrow \infty$ as $t \rightarrow \infty$.

Let $X$ be a linear metric space. A function $p: X \rightarrow \mathbb{R}$ is called paranorm, if

(1) $p(x) \geq 0$, for all $x \in X$,

(2) $p(-x)=p(x)$, for all $x \in X$,

(3) $p(x+y) \leq p(x)+p(y)$, for all $x, y \in X$,

(4) if $\left(\lambda_{n}\right)$ is a sequence of scalars with $\lambda_{n} \rightarrow \lambda$ as $n \rightarrow \infty$ and $\left(x_{n}\right)$ is a sequence of vectors with $p\left(x_{n}-x\right) \rightarrow 0$ as $n \rightarrow \infty$, then $p\left(\lambda_{n} x_{n}-\lambda x\right) \rightarrow 0$ as $n \rightarrow \infty$.

A paranorm $p$ for which $p(x)=0$ implies $x=0$ is called total paranorm and the pair $(X, p)$ is called a total paranormed space. It is well known that the metric of any linear metric space is given by some total paranorm (see [35], Theorem 10.4.2, P-183). For more details about sequence spaces see ([12], [15], [20], [23], [24], [25], [27]) and references therein.

Let $\mathcal{M}=\left(M_{k, l}\right)$ be a sequence of orlicz functions, $p=\left(p_{k, l}\right)$ be a bounded sequence of positive real numbers and $u=\left(u_{k, l}\right)$ be a sequence of strictly positive real numbers. Let $X$ be a seminormed space over the complex field $\mathbb{C}$ with the seminorm $q$. Now we define the following classes of sequences in the present paper:

$$
\begin{gathered}
c^{2}\left(\Delta_{n}^{\mathrm{m}}, \mathcal{M}, \mathrm{u}, p, \mathrm{q}, \mathrm{s}\right)=\left\{x=\left(\mathrm{x}_{\mathrm{k}, \mathrm{l}}\right) \in w^{2}: \mathrm{P}-\lim _{\mathrm{k}, \mathrm{l}}(\mathrm{kl})^{-s} \mathfrak{u}_{\mathrm{k}, \mathrm{l}}\left[\mathrm{M}_{\mathrm{k}, \mathrm{l}}\left(\mathrm{q}\left(\frac{\Delta_{\mathrm{n}}^{\mathrm{m}} \mathrm{x}_{\mathrm{k}, \mathrm{l}}-\mathrm{L}}{\rho}\right)\right)\right]^{\mathrm{p}_{k, \mathrm{l}}}=0,\right. \\
\text { for some } \rho>0, \mathrm{~L} \text { and } \mathrm{s} \geq 0\},
\end{gathered}
$$




$$
\begin{gathered}
\mathrm{c}_{0}^{2}\left(\Delta_{\mathrm{n}}^{\mathrm{m}}, \mathcal{M}, \mathrm{u}, \mathrm{p}, \mathrm{q}, \mathrm{s}\right)=\left\{\mathrm{x}=\left(\mathrm{x}_{\mathrm{k}, \mathrm{l}}\right) \in w^{2}: \mathrm{P}-\lim _{\mathrm{k}, \mathrm{l}}(\mathrm{kl})^{-\mathrm{s}} \mathrm{u}_{\mathrm{k}, \mathrm{l}}\left[\mathrm{M}_{\mathrm{k}, \mathrm{l}}\left(\mathrm{q}\left(\frac{\Delta_{\mathrm{n}}^{\mathrm{m}} \mathrm{x}_{\mathrm{k}, \mathrm{l}}}{\rho}\right)\right)\right]^{\mathrm{p}_{\mathrm{k}, \mathrm{l}}}=0\right. \\
\text { for some } \rho>0 \text { and } \mathrm{s} \geq 0\}
\end{gathered}
$$

and

$$
\begin{gathered}
l_{\infty}^{2}\left(\Delta_{n}^{\mathrm{m}}, \mathcal{M}, \mathrm{u}, \mathrm{p}, \mathrm{q}, \mathrm{s}\right)=\left\{x=\left(\mathrm{x}_{\mathrm{k}, \mathrm{l}}\right) \in w^{2}: \sup _{\mathrm{k}, \mathrm{l}}(\mathrm{kl})^{-\mathrm{s}} \mathrm{u}_{\mathrm{k}, \mathrm{l}}\left[\mathrm{M}_{\mathrm{k}, \mathrm{l}}\left(\mathrm{q}\left(\frac{\Delta_{\mathrm{n}}^{\mathrm{m}} \mathrm{x}_{\mathrm{k}, \mathrm{l}}}{\rho}\right)\right)\right]^{p_{\mathrm{k}, \mathrm{l}}}<\infty\right. \\
\text { for some } \rho>0 \text { and } \mathrm{s} \geq 0\} .
\end{gathered}
$$

If we take $\mathcal{M}(x)=x$, we get

$$
\begin{aligned}
& c^{2}\left(\Delta_{n}^{m}, u, p, q, s\right)=\left\{x=\left(x_{k, l}\right) \in w^{2}: P-\lim _{k, l}(k l)^{-s} u_{k, l}\left[q\left(\frac{\Delta_{n}^{m} x_{k, l}-L}{\rho}\right)\right]^{p_{k, l}}=0,\right. \\
& \text { for some } \rho>0, L \text { and } s \geq 0\} \\
& c_{0}^{2}\left(\Delta_{n}^{m}, u, p, q, s\right)=\left\{x=\left(x_{k, l}\right) \in w^{2}: P-\lim _{k, l}(k l)^{-s} u_{k, l}\left[q\left(\frac{\Delta_{n}^{m} x_{k, l}}{\rho}\right)\right]^{p_{k, l}}=0,\right. \\
& \text { for some } \rho>0 \text { and } s \geq 0\}
\end{aligned}
$$

and

$$
\begin{gathered}
l_{\infty}^{2}\left(\Delta_{n}^{m}, u, p, q, s\right)=\left\{x=\left(x_{k, l}\right) \in w^{2}: \sup _{k, l}(k l)^{-s} u_{k, l}\left[q\left(\frac{\Delta_{n}^{m} x_{k, l}}{\rho}\right)\right]^{p_{k, l}}<\infty\right. \\
\text { for some } \rho>0 \text { and } s \geq 0\}
\end{gathered}
$$

If we take $p=\left(p_{k, l}\right)=1$, we get

$$
\begin{gathered}
c^{2}\left(\Delta_{\mathrm{n}}^{\mathrm{m}}, \mathcal{M}, \mathrm{u}, \mathrm{q}, \mathrm{s}\right)=\left\{x=\left(\mathrm{x}_{\mathrm{k}, \mathrm{l}}\right) \in w^{2}: \mathrm{P}-\lim _{\mathrm{k}, \mathrm{l}}(\mathrm{kl})^{-s} \mathrm{u}_{\mathrm{k}, \mathrm{l}}\left[\mathrm{M}_{\mathrm{k}, \mathrm{l}}\left(\mathrm{q}\left(\frac{\Delta_{\mathrm{n}}^{\mathrm{m}} \mathrm{x}_{\mathrm{k}, \mathrm{l}}-\mathrm{L}}{\rho}\right)\right)\right]=0\right. \\
\text { for some } \rho>0, \mathrm{~L} \text { and } \mathrm{s} \geq 0\} \\
\mathrm{c}_{0}^{2}\left(\Delta_{\mathrm{n}}^{\mathrm{m}}, \mathcal{M}, \mathrm{u}, \mathrm{q}, \mathrm{s}\right)=\left\{x=\left(\mathrm{x}_{\mathrm{k}, \mathrm{l}}\right) \in w^{2}: \mathrm{P}-\lim _{\mathrm{k}, \mathrm{l}}(\mathrm{kl})^{-\mathrm{s}} \mathrm{u}_{\mathrm{k}, \mathrm{l}}\left[\mathrm{M}_{\mathrm{k}, \mathrm{l}}\left(\mathrm{q}\left(\frac{\Delta_{\mathrm{n}}^{\mathrm{m}} \mathrm{x}_{\mathrm{k}, \mathrm{l}}}{\rho}\right)\right)\right]=0\right. \\
\text { for some } \rho>0 \text { and } \mathrm{s} \geq 0\}
\end{gathered}
$$

and

$$
\begin{gathered}
l_{\infty}^{2}\left(\Delta_{n}^{m}, \mathcal{M}, u, q, s\right)=\left\{x=\left(x_{k, l}\right) \in w^{2}: \sup _{k, l}(k l)^{-s} u_{k, l}\left[M_{k, l}\left(q\left(\frac{\Delta_{n}^{m} x_{k, l}}{\rho}\right)\right)\right]<\infty\right. \\
\text { for some } \rho>0 \text { and } s \geq 0\} .
\end{gathered}
$$

If we take $\mathrm{m}=\mathrm{n}=0$ and $\mathrm{q}(\mathrm{x})=|x|$, then we get new double sequence spaces as follows :

$$
c^{2}(\mathcal{M}, u, p, s)=\left\{x=\left(x_{k, l}\right) \in w^{2}: P-\lim _{k, l}(k l)^{-s} u_{k, l}\left[M_{k, l}\left(\frac{\left|x_{k, l}-L\right|}{\rho}\right)\right]^{p_{k, l}}=0\right.
$$




$$
\begin{gathered}
\text { for some } \rho>0, L \text { and } s \geq 0\}, \\
c_{0}^{2}(\mathcal{M}, u, p, s)=\left\{x=\left(x_{k, l}\right) \in w^{2}: P-\lim _{k, l}(k l)^{-s} u_{k, l}\left[M_{k, l}\left(\frac{\left|x_{k, l}\right|}{\rho}\right)\right]^{p_{k, l}}=0,\right. \\
\text { for some } \rho>0 \text { and } s \geq 0\}
\end{gathered}
$$

and

$$
\begin{gathered}
l_{\infty}^{2}(\mathcal{M}, u, p, s)=\left\{x=\left(x_{k, l}\right) \in w^{2}: \sup _{k, l}(k l)^{-s} u_{k, l}\left[M_{k, l}\left(\frac{\left|x_{k, l}\right|}{\rho}\right)\right]^{p_{k, l}}<\infty,\right. \\
\text { for some } \rho>0 \text { and } s \geq 0\} .
\end{gathered}
$$

If we take $\mathrm{m}=\mathrm{n}=1$ and $\mathrm{q}(\mathrm{x})=|x|$, then we get new double sequence spaces as follows :

$$
\begin{gathered}
c^{2}\left(\Delta_{n}^{m}, \mathcal{M}, u, p, s\right)=\left\{x=\left(x_{k, l}\right) \in w^{2}: P-\lim _{k, l}(k l)^{-s} u_{k, l}\left[M_{k, l}\left(\frac{\left|\Delta x_{k, l}-L\right|}{\rho}\right)\right]^{p_{k, l}}=0,\right. \\
\text { for some } \rho>0, L \text { and } s \geq 0\} \\
c_{0}^{2}\left(\Delta_{n}^{m}, \mathcal{M}, u, p, s\right)=\left\{x=\left(x_{k, l}\right) \in w^{2}: P-\lim _{k, l}(k l)^{-s} u_{k, l}\left[M_{k, l}\left(\frac{\left|\Delta x_{k, l}\right|}{\rho}\right)\right]^{p_{k, l}}=0,\right. \\
\text { for some } \rho>0 \text { and } s \geq 0\}
\end{gathered}
$$

and

$$
\begin{gathered}
l_{\infty}^{2}\left(\Delta_{n}^{m}, \mathcal{M}, u, p, s\right)=\left\{x=\left(x_{k, l}\right) \in w^{2}: \sup _{k, l}(k l)^{-s} u_{k, l}\left[M_{k, l}\left(\frac{\left|\Delta_{n}^{m} x_{k, l}\right|}{\rho}\right)\right]^{p_{k, l}}<\infty,\right. \\
\text { for some } \rho>0 \text { and } s \geq 0\} .
\end{gathered}
$$

The following inequality will be used throughout the paper. Let $p=\left(p_{k, l}\right)$ be a double sequence of positive real numbers with $0<p_{k, l} \leq \sup _{k, l}=H$ and let $\mathrm{K}=\max \left\{1,2^{\mathrm{H}-1}\right\}$. Then for the factorable sequences $\left\{a_{k, l}\right\}$ and $\left\{b_{k, l}\right\}$ in the complex plane, we have

$$
\left|a_{k, l}+b_{k, l}\right|^{p_{k, l}} \leq K\left(\left|a_{k, l}\right|^{p_{k, l}}+\left|b_{k, l}\right|^{p_{k, l}}\right) .
$$

The main goal of this paper is to extend a few known results in the literature from single difference sequence spaces to double difference sequence spaces. We also make an effort to study some topological properties and inclusion relations between above defined sequence spaces. 


\section{$2 \quad$ Main Results}

Theorem 2.1 Let $\mathcal{M}=\left(M_{k, l}\right)$ be a sequence of Orlicz functions, $p=\left(p_{k, l}\right)$ be a bounded sequence of positive real numbers and $\mathfrak{u}=\left(\mathfrak{u}_{k, l}\right)$ be a sequence of strictly positive real numbers, then the classes of sequences $\mathrm{c}_{\mathrm{o}}^{2}\left(\Delta_{\mathfrak{n}}^{\mathrm{m}}, \mathcal{M}, \mathrm{u}, \mathrm{p}, \mathrm{q}, \mathrm{s}\right), \mathrm{c}^{2}\left(\Delta_{\mathfrak{n}}^{\mathrm{m}}, \mathcal{M}, \mathrm{u}, \mathrm{p}, \mathrm{q}, \mathrm{s}\right)$ and $\mathrm{l}_{\infty}\left(\Delta_{\mathfrak{n}}^{\mathrm{m}}, \mathcal{M}, \mathrm{u}, \mathrm{p}, \mathrm{q}, \mathrm{s}\right)$ are linear spaces over the field of complex numbers $\mathbb{C}$.

Proof. Let $x=\left(x_{k, l}\right), y=\left(y_{k, l}\right) \in c_{0}^{2}\left(\Delta_{\mathfrak{n}}^{m}, \mathcal{M}, \mathfrak{u}, p, q, s\right)$ and $\alpha, \beta \in \mathbb{C}$. Then there exist positive numbers $\rho_{1}$ and $\rho_{2}$ such that

$$
\lim _{k, l}(k l)^{-s} u_{k, l}\left[M_{k, l}\left(q\left(\frac{\Delta_{n}^{m} x_{k, l}}{\rho_{1}}\right)\right)\right]^{p_{k, l}}=0, \text { for some } \rho_{1}>0
$$

and

$$
\lim _{k, l}(k l)^{-s} u_{k, l}\left[M_{k, l}\left(q\left(\frac{\Delta_{m}^{n} y_{k, l}}{\rho_{2}}\right)\right)\right]^{p_{k, l}}=0, \text { for some } \rho_{2}>0 .
$$

Let $\rho_{3}=\max \left(2|\alpha| \rho_{1}, 2|\beta| \rho_{2}\right)$. Since $\mathcal{M}=\left(M_{k, l}\right)$ is non-decreasing convex function and so by using inequality (1.1), we have

$$
\begin{aligned}
\lim _{k, l}(k l)^{-s} u_{k, l}\left[M_{k, l}\right. & \left.\left(q\left(\frac{\Delta_{n}^{m}\left(\alpha x_{k, l}+\beta y_{k, l}\right)}{\rho_{3}}\right)\right)\right]^{p_{k, l}} \\
& =\lim _{k, l}(k l)^{-s} u_{k, l}\left[M_{k, l}\left(q\left(\frac{\alpha \Delta_{n}^{m} x_{k, l}}{\rho_{3}}\right)+q\left(\frac{\beta \Delta_{n}^{m} y_{k, l}}{\rho_{3}}\right)\right)\right]^{p_{k, l}} \\
& \leq K \lim _{k, l} \frac{1}{2^{p_{k, l}}}(k l)^{-s} u_{k, l}\left[M_{k, l}\left(q\left(\frac{\Delta_{n}^{m} x_{k, l}}{\rho_{1}}\right)\right)\right]^{p_{k, l}} \\
& +K \lim _{k, l} \frac{1}{2^{p_{k, l}}}(k l)^{-s} u_{k, l}\left[M_{k, l}\left(q\left(\frac{\Delta_{n}^{m} y_{k, l}}{\rho_{2}}\right)\right)\right]^{p_{k, l}} \\
& \leq K \lim _{k, l}(k l)^{-s} u_{k, l}\left[M_{k, l}\left(q\left(\frac{\Delta_{n}^{m} x_{k, l}}{\rho_{1}}\right)\right)\right]^{p_{k, l}} \\
& +K \lim _{k, l}(k l)^{-s} u_{k, l}\left[M_{k, l}\left(q\left(\frac{\Delta_{n}^{m} y_{k, l}}{\rho_{2}}\right)\right)\right]^{p_{k, l}} \\
& =0 .
\end{aligned}
$$

So, $\alpha x+\beta y \in c_{0}^{2}\left(\Delta_{m}^{n}, \mathcal{M}, u, p, q, s\right)$. Hence $c_{0}^{2}\left(\Delta_{m}^{n}, \mathcal{M}, u, p, q, s\right)$ is a linear space. Similarly, we can prove that $\mathrm{c}^{2}\left(\Delta_{\mathrm{m}}^{\mathrm{n}}, \mathcal{M}, \mathrm{u}, \mathrm{p}, \mathrm{q}, \mathrm{s}\right)$ and $\mathrm{l}_{\infty}^{2}\left(\Delta_{\mathrm{m}}^{\mathrm{n}}, \mathcal{M}, \mathrm{u}, \mathrm{p}, \mathrm{q}, \mathrm{s}\right)$ are linear spaces.

Theorem 2.2 Let $\mathcal{M}=\left(M_{k, l}\right)$ be a sequence of Orlicz functions, $p=\left(p_{k, l}\right)$ be a bounded sequence of positive real numbers and $\mathfrak{u}=\left(\mathfrak{u}_{k, l}\right)$ be a sequence of strictly positive real numbers. For $\mathrm{Z}^{2}=\mathrm{l}_{\infty}^{2}, \mathrm{c}^{2}$ and $\mathrm{c}_{0}^{2}$, the spaces $\mathrm{Z}^{2}\left(\Delta_{\mathrm{n}}^{\mathrm{m}}, \mathcal{M}, \mathrm{u}, \mathrm{p}, \mathrm{q}, \mathrm{s}\right)$ are paranormed spaces, paranormed by

$$
g(x)=\sum_{k, l=1}^{n m} q\left(x_{k, l}\right)+\inf \left\{\rho^{\frac{p_{k, l}}{H}}: \sup _{k, l}(k l)^{-s} u_{k, l} M_{k, l}\left(q\left(\frac{\Delta_{n}^{m} x_{k, l}}{\rho}\right)\right) \leq 1\right\}
$$

where $\mathrm{H}=\max \left(1, \sup _{k, \mathrm{l}} \mathrm{p}_{\mathrm{k}, \mathrm{l}}\right)$. 
Proof. Clearly $g(-x)=g(x), g(0)=0$. Let $\left(x_{k, l}\right)$ and $\left(y_{k, l}\right)$ be any two sequences belong to any one of the spaces $Z^{2}\left(\Delta_{m}^{n}, \mathcal{M}, u, p, q, s\right)$, for $Z^{2}=c_{0}^{2}, c^{2}$ and $l_{\infty}^{2}$. Then, we get $\rho_{1}, \rho_{2}>0$ such that

$$
\sup _{k, l}(k l)^{-s} u_{k, l} M_{k, l}\left(q\left(\frac{\Delta_{n}^{m} x_{k, l}}{\rho_{1}}\right)\right) \leq 1
$$

and

$$
\sup _{k, l}(k l)^{-s} u_{k, l} M_{k, l}\left(q\left(\frac{\left.\Delta_{n}^{m} y_{k, l}\right)}{\rho_{2}}\right) \leq 1 .\right.
$$

Let $\rho=\rho_{1}+\rho_{2}$. Then by convexity of $\mathcal{M}=\left(M_{k, l}\right)$, we have

$$
\begin{aligned}
\sup _{k, l}(k l)^{-s} u_{k, l} M_{k, l}\left(q\left(\frac{\Delta_{n}^{m}\left(x_{k, l}+y_{k, l}\right)}{\rho}\right)\right) & \leq\left(\frac{\rho_{1}}{\rho_{1}+\rho_{2}}\right) \sup _{k, l}(k l)^{-s} u_{k, l} M_{k, l}\left(q\left(\frac{\Delta_{n}^{m} x_{k, l}}{\rho_{1}}\right)\right) \\
& +\left(\frac{\rho_{2}}{\rho_{1}+\rho_{2}}\right) \sup _{k, l}(k l)^{-s} u_{k, l} M_{k, l}\left(q\left(\frac{\Delta_{n}^{m} y_{k, l}}{\rho_{2}}\right)\right) \\
& \leq 1 .
\end{aligned}
$$

Hence we have,

$$
\begin{aligned}
g(x+y) & =\sum_{k, l=1}^{m n} q\left(x_{k, l}+y_{k, l}\right) \\
& +\inf \left\{\rho^{\frac{p_{k}, l}{H}}: \sup _{k, l}(k l)^{-s} u_{k, l} M_{k, l}\left(q\left(\frac{\Delta_{n}^{m}\left(x_{k, l}+y_{k, l}\right)}{\rho}\right)\right) \leq 1\right\} \\
& \leq \sum_{k, l=1}^{m n} q\left(x_{k, l}\right)+\inf \left\{\rho_{1}^{\frac{p_{k}, l}{H}}: \sup _{k, l}(k l)^{-s} u_{k, l} M_{k, l}\left(q\left(\frac{\Delta_{n}^{m} x_{k, l}}{\rho_{1}}\right)\right) \leq 1\right\} \\
& +\sum_{k, l=1}^{m n} q\left(y_{k, l}\right)+\inf \left\{\rho_{2}^{\frac{p_{k}, l}{H}}: \sup _{k, l}(k l)^{-s} u_{k, l} M_{k, l}\left(q\left(\frac{\Delta_{n}^{m} y_{k, l}}{\rho_{2}}\right)\right) \leq 1\right\} .
\end{aligned}
$$

This implies that

$$
g(x+y) \leq g(x)+g(y) .
$$

The continuity of the scalar multiplication follows from the following inequality

$$
\begin{aligned}
g(\mu x) & =\sum_{k, l=1}^{m n} q\left(\mu x_{k, l}\right)+\inf \left\{\rho^{\frac{p_{k, l} l}{H}}: \sup _{k, l}(k l)^{-s} u_{k, l} M_{k, l}\left(q\left(\frac{\Delta_{n}^{m} \mu x_{k, l}}{\rho}\right)\right) \leq 1\right\} \\
& =|\mu| \sum_{k, l=1}^{m n} q\left(x_{k, l}\right)+\inf \left\{(t|\mu|)^{\frac{p_{k}, l}{H}}: \sup _{k, l}(k l)^{-s} u_{k, l} M_{k, l}\left(q\left(\frac{\Delta_{n}^{m} x_{k, l}}{t}\right)\right) \leq 1\right\},
\end{aligned}
$$

where $t=\frac{\rho}{|\mu|}$. Hence the space $Z^{2}\left(\Delta_{m}^{n}, \mathcal{M}, u, p, q, s\right)$, for $Z^{2}=c_{0}^{2}, c^{2}$ and $l_{\infty}^{2}$ is a paranormed space, paranormed by $g$.

Theorem 2.3 Let $\mathcal{M}=\left(M_{k, l}\right)$ be a sequence of Orlicz functions, $\mathrm{p}=\left(\mathrm{p}_{\mathrm{k}, \mathrm{l}}\right)$ be a bounded sequence of positive real numbers and $\mathfrak{u}=\left(\mathfrak{u}_{\mathrm{k}, \mathrm{l}}\right)$ be a sequence of strictly positive real numbers. 
For $\mathrm{Z}^{2}=\mathrm{l}_{\infty}^{2}, \mathrm{c}^{2}$ and $\mathrm{c}_{0}^{2}$, the spaces $\mathrm{Z}^{2}\left(\Delta_{\mathrm{n}}^{\mathrm{m}}, \mathcal{M}, \mathrm{u}, \mathrm{p}, \mathrm{q}, \mathrm{s}\right)$ are complete paranormed spaces, paranormed by

$$
g(x)=\sum_{k, l=1}^{n m} q\left(x_{k, l}\right)+\inf \left\{\rho^{\frac{p_{k}, l}{H}}: \sup _{k, l}(k l)^{-s} u_{k, l} M_{k, l}\left(q\left(\frac{\Delta_{n}^{m} x_{k, l}}{\rho}\right)\right) \leq 1\right\}
$$

where $\mathrm{H}=\max \left(1, \sup _{\mathrm{k}, \mathrm{l}} \mathrm{p}_{\mathrm{k}, \mathrm{l}}\right)$.

Proof. We prove the result for the space $l_{\infty}^{2}\left(\Delta_{n}^{m}, \mathcal{M}, u, p, q, s\right)$. Let $\left(x_{k, l}^{i}\right)$ be any Cauchy sequence in $l_{\infty}^{2}\left(\Delta_{n}^{m}, \mathcal{M}, u, p, q, s\right)$. Let $\epsilon>0$ be given and for $t>0$, choose $x_{0}$ be fixed such that $u_{k, l} M_{k, l}\left(\frac{t x_{0}}{2}\right) \geq 1$, then there exists a positive integer $n_{0} \in \mathbb{N}$ such that $g\left(x_{k, l}^{i}-x_{k, l}^{j}\right)<\frac{\epsilon}{x_{0} t}$, for all $i, j \geq n_{0}$. Using the definition of paranorm, we get

$$
\begin{gathered}
\sum_{k, l=1}^{m n} q\left(x_{k, l}^{i}-x_{k, l}^{j}\right)+\inf \left\{\rho^{\frac{p_{k, l}}{H}}: \sup _{k, l}(k l)^{-s} u_{k, l} M_{k, l}\left(q\left(\frac{\Delta_{n}^{m}\left(x_{k, l}^{i}-x_{k, l}^{j}\right.}{\rho}\right)\right)\right\}<\frac{\epsilon}{x_{0} t} \\
\text { for all } i, j \geq n_{0}
\end{gathered}
$$

Hence we have,

$$
\sum_{k, l=1}^{m n} q\left(x_{k, l}^{i}-x_{k, l}^{j}\right)<\epsilon, \text { for all } i, j \geq n_{0}
$$

This implies that

$$
q\left(x_{k, l}^{i}-x_{k, l}^{j}\right)<\epsilon, \text { for all } i, j \geq n_{0} \text { and } 1 \leq k \leq n m
$$

Thus $\left(x_{k, l}^{i}\right)$ is a Cauchy sequence in $\mathbb{C}$ for $k, l=1,2, \ldots, n m$. Hence $\left(x_{k, l}^{i}\right)$ is convergent in $\mathbb{C}$ for $k, l=1,2, \ldots ., n m$. Let $\lim _{i \rightarrow \infty} x_{k, l}^{i}=x_{k, l}$, say for $k, l=1,2, \ldots, n m$.

Again from equation (2.1) we have,

$$
\inf \left\{\rho^{\frac{p_{k, l}}{H}}: \sup _{k, l}(k l)^{-s} u_{k, l} M_{k, l}\left(q\left(\frac{\Delta_{n}^{m}\left(x_{k, l}^{i}-x_{k, l}^{j}\right)}{\rho}\right)\right) \leq 1\right\}<\epsilon, \text { for all } i, j \geq n_{0} .
$$

Hence we get

$$
\sup _{k, l}(k l)^{-s} u_{k, l} M_{k, l}\left(q\left(\frac{\Delta_{n}^{m}\left(x_{k, l}^{i}-x_{k, l}^{j}\right)}{g\left(x^{i}-x^{j}\right)}\right)\right) \leq 1, \text { for all } i, j \geq n_{0} .
$$

It follows that $(k l)^{-s} u_{k, l} M_{k, l}\left(q\left(\frac{\Delta_{n}^{m}\left(x_{k, l}^{i}-x_{k, l}^{j}\right)}{g\left(x^{i}-x^{j}\right)}\right)\right) \leq 1$, for each $k, l \geq 1$ and for all $i, j \geq n_{0}$.

For $t>0$ with $(k l)^{-s} u_{k, l} M_{k, l}\left(\frac{t x_{0}}{2}\right) \geq 1$, we have

$$
(k l)^{-s} u_{k, l} M_{k, l}\left(q\left(\frac{\Delta_{n}^{m}\left(x_{k, l}^{i}-x_{k, l}^{j}\right)}{g\left(x^{i}-x^{j}\right)}\right)\right) \leq(k l)^{-s} u_{k, l} M_{k, l}\left(\frac{t x_{0}}{2}\right)
$$

This implies that

$$
q\left(\Delta_{n}^{m} x_{k, l}^{i}-\Delta_{n}^{m} x_{k, l}^{j}\right)<\frac{t x_{0}}{2} \frac{\epsilon}{t x_{0}}=\frac{\epsilon}{2} .
$$


Hence $\mathrm{q}\left(\Delta_{\mathrm{n}}^{\mathrm{m}} x_{k, l}^{\mathrm{i}}\right)$ is a Cauchy sequence in $\mathbb{C}$ for all $k, l \in \mathbb{N}$. This implies that $\mathrm{q}\left(\Delta_{\mathrm{n}}^{\mathrm{m}} x_{k, l}^{i}\right)$ is convergent in $\mathbb{C}$ for all $k, l \in \mathbb{N}$. Let $\lim _{i \rightarrow \infty} q\left(\Delta_{n}^{m} x_{k, l}^{i}\right)=y_{k, l}$ for each $k, l \in \mathbb{N}$. Let $k, l=1$, then we have

$$
\lim _{i \rightarrow \infty} \mathrm{q}\left(\Delta_{\mathrm{n}}^{\mathrm{m}} \mathrm{x}_{1,1}^{\mathrm{i}}\right)=\lim _{i \rightarrow \infty} \sum_{v=0}^{\mathrm{m}}(-1)^{v}\left(\begin{array}{c}
\mathrm{m} \\
v
\end{array}\right) x_{1+n v, 1+\mathrm{m} v}^{i}=\mathrm{y}_{1,1} .
$$

We have by equation (2.2) and equation (2.3) $\lim _{i \rightarrow \infty} x_{m n+1}^{i}=x_{m n+1}$, exists. Proceeding in this way inductively, we have $\lim _{i \rightarrow \infty} x_{k, l}^{i}=x_{k, l}$ exists for each $k, l \in \mathbb{N}$. Now we have for all $i, j \geq n_{0}$,

$$
\sum_{k, l=1}^{m n} q\left(x_{k, l}^{i}-x_{k, l}^{j}\right)+\inf \left\{\rho^{\frac{p_{k, l}}{H}}: \sup _{k, l}(k l)^{-s} u_{k, l} M_{k, l}\left(q\left(\frac{\Delta_{n}^{m}\left(x_{k, l}^{i}-x_{k, l}^{j}\right)}{\rho}\right)\right) \leq 1\right\}<\epsilon .
$$

This implies that

$$
\lim _{j \rightarrow \infty}\left\{\sum_{k, l=1}^{m n} q\left(x_{k, l}^{i}-x_{k, l}^{j}\right)+\inf \left\{\rho^{\frac{p_{k, l}}{H}}: \sup _{k, l}(k l)^{-s} u_{k, l} M_{k, l}\left(q\left(\frac{\Delta_{n}^{m}\left(x_{k, l}^{i}-x_{k, l}^{j}\right)}{\rho}\right)\right) \leq 1\right\}\right\}<\epsilon,
$$

for all $i \geq n_{0}$. Using the continuity of $M_{k, l}$, we have

$$
\sum_{k, l=1}^{m n} q\left(x_{k, l}^{i}-x_{k, l}\right)+\inf \left\{\rho^{\frac{p_{k, l}}{H}}: \sup _{k, l}(k l)^{-s} u_{k, l} M_{k, l}\left(q\left(\frac{\Delta_{n}^{m} x_{k, l}^{i}-\Delta_{n}^{m} x_{k, l}}{\rho}\right)\right) \leq 1\right\}<\epsilon,
$$

for all $i \geq n_{0}$. It follows that $\left(\chi^{i}-x\right) \in l_{\infty}^{2}\left(\Delta_{n}^{m}, \mathcal{M}, u, p, q, s\right)$. Since $x^{i} \in l_{\infty}^{2}\left(\Delta_{m}^{n}, \mathcal{M}, u, p, q, s\right)$ and $l_{\infty}^{2}\left(\Delta_{\mathfrak{n}}^{\mathrm{m}}, \mathcal{M}, \mathrm{u}, \mathrm{p}, \mathrm{q}, \mathrm{s}\right)$ is a linear space, so we have $x=x^{i}-\left(x^{i}-x\right) \in l_{\infty}^{2}\left(\Delta_{\mathrm{m}}^{\mathrm{n}}, \mathcal{M}, \mathrm{u}, \mathrm{p}, \mathrm{q}, \mathrm{s}\right)$. This completes the proof. Similarly, we can prove that $c^{2}\left(\Delta_{m}^{n}, \mathcal{M}, u, p, q, s\right)$ and $c_{0}^{2}\left(\Delta_{m}^{n}, \mathcal{M}, u, p, q, s\right)$ are complete paranormed spaces in view of the above proof.

Theorem 2.4 Let $\mathrm{m} \geq 1$, then for all $0<i \leq \mathrm{m}, \mathrm{Z}^{2}\left(\Delta_{\mathfrak{n}}^{\mathrm{i}}, \mathcal{M}, \mathrm{u}, \mathrm{p}, \mathrm{q}, \mathrm{s}\right) \subset \mathrm{Z}^{2}\left(\Delta_{\mathfrak{n}}^{\mathrm{m}}, \mathcal{M}, \mathrm{u}, \mathrm{p}, \mathrm{q}, \mathrm{s}\right)$, where $Z^{2}=c^{2}, c_{0}^{2}$ and $l_{\infty}^{2}$.

Proof. We will prove it for only $c_{0}^{2}\left(\Delta_{n}^{m-1}, \mathcal{M}, u, p, q, s\right)$. Let $x=\left(x_{k, l}\right) \in c_{0}^{2}\left(\Delta_{n}^{m-1}, \mathcal{M}, u, p, q, s\right)$. Then

$$
P-\lim _{k, l}(k l)^{-s} \mathfrak{u}_{k, l}\left[M_{k, l}\left(\frac{q\left(\Delta_{n}^{m-1} \chi_{k, l}\right)}{\rho}\right)\right]^{P_{k, l}}=0, \text { for some } \rho>0 \text { and } s \geq 0
$$

Then from (2.4) we have

$$
\begin{aligned}
& P-\lim _{k, l}(k l)^{-s} u_{k, l}\left[M_{k, l}\left(q\left(\frac{\Delta_{n}^{m} x_{k, l}}{\rho}\right)\right)\right]^{P_{k, l+1}}=0, \\
& P-\lim _{k, l}(k l)^{-s} u_{k, l}\left[M_{k, l}\left(q\left(\frac{\Delta_{n}^{m} x_{k, l}}{\rho}\right)\right)\right]^{P_{k+1, l}}=0
\end{aligned}
$$

and

$$
\mathrm{P}-\lim _{\mathrm{k}, \mathrm{l}}(\mathrm{kl})^{-s} \mathfrak{u}_{k, l}\left[M_{k, l}\left(\mathrm{q}\left(\frac{\Delta_{\mathrm{n}}^{\mathrm{m}} \boldsymbol{\chi}_{k, l}}{\rho}\right)\right)\right]^{\mathrm{P}_{\mathrm{k}+1, l+1}}=0 .
$$


Now for

$$
\Delta_{n}^{m} \chi=\left(\Delta_{n}^{m} \chi_{k, l}\right)=\left(\Delta_{n}^{m-1} \chi_{k, l}-\Delta_{n}^{m-1} \chi_{k, l+1}-\Delta_{n}^{m-1} \chi_{k+1, l}+\Delta_{n}^{m-1} \chi_{k+1, l+1}\right),
$$

we have

$$
\begin{aligned}
& (k l)^{-s} u_{k, l}\left[M_{k, l}\left(q\left(\frac{\Delta_{n}^{m} x_{k, l}}{\rho}\right)\right)\right]^{P_{k, l}}
\end{aligned}
$$

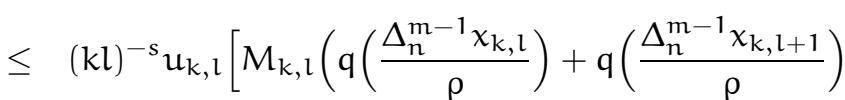

$$
\begin{aligned}
& \left.\left.+q\left(\frac{\Delta_{n}^{m-1} x_{k+1, l}}{\rho}\right)+q\left(\frac{\Delta_{n}^{m-1} x_{k+1, l+1}}{\rho}\right)\right)\right]^{P_{k, l}} \\
& \leq \mathrm{K}^{2}(\mathrm{kl})^{-s} \mathrm{u}_{\mathrm{k}, \mathrm{l}}\left\{\left[M_{k, l}\left(\mathrm{q}\left(\frac{\Delta_{\mathrm{n}}^{\mathrm{m}-1} \mathrm{x}_{\mathrm{k}, \mathrm{l}}}{\rho}\right)\right)\right]^{\mathrm{P}_{\mathrm{k}, \mathrm{l}}}+\mathrm{u}_{\mathrm{k}, \mathrm{l}}\left[\mathrm{M}_{\mathrm{k}, \mathrm{l}}\left(\mathrm{q}\left(\frac{\Delta_{\mathrm{n}}^{\mathrm{m}-1} \chi_{\mathrm{k}+1, \mathrm{l}}}{\rho}\right)\right)\right]^{\mathrm{P}_{k, l}}\right. \\
& \left.+u_{k, l}\left[M\left(q\left(\frac{\Delta_{n}^{m-1} x_{k, l+1}}{\rho}\right)\right)\right]^{P_{k, l}}+u_{k, l}\left[M_{k, l}\left(q\left(\frac{\Delta_{n}^{m-1} \chi_{k+1, l+1}}{\rho}\right)\right)\right]^{P_{k, l}}\right\}
\end{aligned}
$$

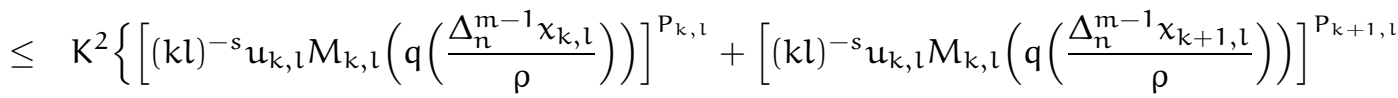

$$
\begin{aligned}
& \left.+\left[(k l)^{-s} u_{k, l} M_{k, l}\left(q\left(\frac{\Delta_{n}^{m-1} x_{k, l+1}}{\rho}\right)\right)\right]^{P_{k, l+1}}+u_{k, l}\left[(k l)^{-s} M_{k, l}\left(q\left(\frac{\Delta_{n}^{m-1} x_{k+1, l+1}}{\rho}\right)\right)\right]^{P_{k+1, l+1}}\right\}
\end{aligned}
$$

from this it follows that $x=\left(x_{k, l}\right) \in c_{0}^{2}\left(\Delta_{n}^{m}, \mathcal{M}, u, p, q, s\right)$ and hence $c_{0}^{2}\left(\Delta_{n}^{m-1}, \mathcal{M}, u, p, q, s\right) \subset$ $\mathrm{c}_{0}^{2}\left(\Delta_{\mathrm{n}}^{\mathrm{m}}, \mathcal{M}, \mathrm{u}, \mathrm{p}, \mathrm{q}, \mathrm{s}\right)$. On applying the principle of induction, it follows that $\mathrm{c}_{\mathrm{O}}^{2}\left(\Delta_{\mathrm{n}}^{\mathrm{i}}, \mathcal{M}, \mathrm{u}, \mathrm{p}, \mathrm{q}, \mathrm{s}\right) \subset$ $c_{0}^{2}\left(\Delta_{n}^{m}, \mathcal{M}, u, p, q, s\right)$ for $i=0,1,2, \cdots, m-1$. Similarly, we can prove the other cases.

Theorem 2.5 (a) If $0<\inf _{k, l} p_{k, l} \leq p_{k, l}<1$, then $Z^{2}\left(\Delta_{n}^{m}, \mathcal{M}, u, p, q, s\right) \subset Z^{2}\left(\Delta_{n}^{m}, \mathcal{M}, u, q, s\right)$, (b) If $1<p_{k, l} \leq \sup _{k, l} p_{k, l}<\infty$, then $Z^{2}\left(\Delta_{n}^{m}, \mathcal{M}, u, q, s\right) \subset Z^{2}\left(\Delta_{n}^{m}, \mathcal{M}, u, p, q, s\right)$, where $\mathrm{Z}^{2}=\mathrm{c}^{2}, \mathrm{c}_{0}^{2}$ and $\mathrm{l}_{\infty}^{2}$.

Proof. (i) Let $x=\left(x_{k, l}\right) \in l_{\infty}^{2}\left(\Delta_{n}^{m}, \mathcal{M}, u, p, q, s\right)$. Since $0<\inf p_{k, l} \leq 1$, we have

$$
\sup _{k, l}(k l)^{-s} u_{k, l}\left[M_{k, l}\left(q\left(\frac{\Delta_{n}^{m} x_{k, l}}{\rho}\right)\right)\right] \leq \sup _{k, l}(k l)^{-s} u_{k, l}\left[M_{k, l}\left(q\left(\frac{\Delta_{n}^{m} x_{k, l}}{\rho}\right)\right)\right]^{p_{k, l}},
$$

and hence $x=\left(x_{k, l}\right) \in l_{\infty}^{2}\left(\Delta_{n}^{m}, \mathcal{M}, u, p, q, s\right)$.

(ii) Let $p_{k, l}$ for each $(k, l)$ and $\sup _{k, l} p_{k, l}<\infty$. Let $x=\left(x_{k, l}\right) \in l_{\infty}^{2}\left(\Delta_{n}^{m}, \mathcal{M}, u, q, s\right)$. Then, for each $0<\epsilon<1$, there exists a positive integer $\mathbb{N}$ such that

$$
\sup _{k, l}(k l)^{-s} u_{k, l}\left[M_{k, l}\left(q\left(\frac{\Delta_{n}^{m} x_{k, l}}{\rho}\right)\right)\right] \leq \epsilon<1,
$$

for all $m, n \in \mathbb{N}$. This implies that

$$
\sup _{k, l}(k l)^{-s} u_{k, l}\left[M_{k, l}\left(q\left(\frac{\Delta_{n}^{m} x_{k, l}}{\rho}\right)\right)\right]^{p_{k, l}} \leq \sup _{k, l}(k l)^{-s} u_{k, l}\left[M_{k, l}\left(q\left(\frac{\Delta_{n}^{m} x_{k, l}}{\rho}\right)\right)\right] .
$$


Thus $x=\left(x_{k, l}\right) \in l_{\infty}^{2}\left(\Delta_{n}^{m}, \mathcal{M}, u, p, q, s\right)$ and this completes the proof.

Theorem 2.6 Let $\mathcal{M}^{\prime}=\left(M_{k, l}^{\prime}\right)$ and $\mathcal{M}^{\prime \prime}=\left(M_{k, l}^{\prime \prime}\right)$ be two sequences of Orlicz functions satisfying $\Delta_{2}$-condition. If $\beta=\lim _{\mathrm{t} \rightarrow \infty} \frac{M_{\mathrm{k}, \mathrm{l}}^{\prime \prime}(\mathrm{t})}{\mathrm{t}} \geq 1$, then $\mathrm{Z}^{2}\left(\Delta_{\mathrm{n}}^{\mathrm{m}}, \mathcal{M}^{\prime}, \mathrm{u}, \mathrm{p}, \mathrm{q}, \mathrm{s}\right)=\mathrm{Z}^{2}\left(\Delta_{\mathrm{n}}^{\mathrm{m}}, \mathcal{M}^{\prime \prime} \circ \mathcal{M}^{\prime}, \mathrm{u}, \mathrm{p}, \mathrm{q}, \mathrm{s}\right)$, where $Z^{2}=c^{2}, c_{0}^{2}$ and $l_{\infty}^{2}$.

Proof. We prove it for $Z^{2}=c^{2}$ and the other cases will follows on applying similar techniques. Let $x=\left(x_{k, l}\right) \in c^{2}\left(\Delta_{n}^{m}, \mathcal{M}^{\prime}, u, p, q, s\right)$, then

$$
\mathrm{P}-\lim _{\mathrm{k}, \mathrm{l}}(\mathrm{kl})^{-\mathrm{s}}\left[\mathrm{M}_{\mathrm{k}, \mathrm{l}}^{\prime}\left(\mathrm{q}\left(\frac{\Delta_{\mathrm{n}}^{\mathrm{m}} \mathrm{x}_{\mathrm{k}, \mathrm{l}}-\mathrm{L}}{\rho}\right)\right)\right]^{\mathrm{P}_{\mathrm{k}, \mathrm{l}}}=0 .
$$

Let $0<\epsilon<1$ and $\delta$ with $0<\delta<1$ such that $M_{k, l}^{\prime \prime}(t)<\epsilon$ for $0 \leq t<\delta$. Let

$$
y_{k, l}=M_{k, l}^{\prime}\left(q\left(\frac{\Delta_{n}^{m} x_{k, l}-L}{\rho}\right)\right)
$$

and consider

$$
\left[M_{k, l}^{\prime \prime}\left(y_{k, l}\right)\right]^{p_{k, l}}=\left[M_{k, l}^{\prime \prime}\left(y_{k, l}\right)\right]^{p_{k, l}}+\left[M_{k, l}^{\prime \prime}\left(y_{k, l}\right)\right]^{p_{k, l}}
$$

where the first term is over $y_{k, l} \leq \delta$ and the second is over $y_{k, l}>\delta$. From the first term in (2.5), we have

$$
(k l)^{-s}\left[M_{k, l}^{\prime \prime}\left(y_{k, l}\right)\right]^{p_{k, l}}<(k l)^{-s}\left[M_{k, l}^{\prime \prime}(2)\right]^{\mathrm{H}}\left[\left(y_{k, l}\right)\right]^{p_{k, l}}
$$

On the other hand, we use the fact that

$$
y_{k, l}<\frac{y_{k, l}}{\delta}<1+\frac{y_{k, l}}{\delta} .
$$

Since $\left(M_{k, l}^{\prime \prime}\right)$ for each $k, l$ is non-decreasing and convex, it follows that

$$
M_{k, l}^{\prime \prime}\left(y_{k, l}\right)<M_{k, l}^{\prime \prime}\left(1+\frac{y_{k, l}}{\delta}\right)<\frac{1}{2} M_{k, l}^{\prime \prime}(2)+\frac{1}{2} M_{k, l}^{\prime \prime}\left(\frac{2 y_{k, l}}{\delta}\right) .
$$

Since $\left(M_{k, l}^{\prime \prime}\right)$ for each $k, l$ satisfies $\Delta_{2}$-condition, we have

$$
M_{k, l}^{\prime \prime}\left(y_{k, l}\right)<\frac{1}{2} K \frac{y_{k, l}}{\delta} M_{k, l}^{\prime \prime}(2)+\frac{1}{2} K \frac{y_{k, l}}{\delta} M_{k, l}^{\prime \prime}(2)=K \frac{y_{k, l}}{\delta} M_{k, l}^{\prime \prime}(2) .
$$

Hence, from the second term in (2.5), it follows that

$$
(k l)^{-s}\left[M^{\prime \prime}\left(y_{k, l}\right)\right]^{p_{k, l}} \leq \max \left(1,\left(K M^{\prime \prime}(2) \delta^{-1}\right)^{H}\right)(k l)^{-s}\left[\left(y_{k, l}\right)\right]^{p_{k, l}}
$$

By the inequalities (2.6) and (2.7), taking limit in the Pringsheim sense, we have $x=\left(x_{k, l}\right) \in$ $c^{2}\left(\Delta_{n}^{m}, \mathcal{M}^{\prime \prime} \circ \mathcal{M}^{\prime}, u, p, q, s\right)$. Observe that in this part of the proof we did not need $\beta \geq 1$. Now, let $\beta \geq 1$ and $x=\left(x_{k, l}\right) \in c^{2}\left(\mathcal{M}^{\prime}, \Delta_{n}^{r}, u, q, p\right)$. Then, we have $M_{k, l}^{\prime \prime}(t) \geq \beta(t)$ for all $t \geq 0$. It follows that $x=\left(x_{k, l}\right) \in c^{2}\left(\Delta_{n}^{m}, \mathcal{M}^{\prime \prime} \circ \mathcal{M}^{\prime}, u, p, q, s\right)$ implies $x=\left(x_{k, l}\right) \in c^{2}\left(\Delta_{n}^{m}, \mathcal{M}^{\prime}, u, p, q, s\right)$. 
This implies $c^{2}\left(\Delta_{\mathrm{n}}^{\mathrm{m}}, \mathcal{M}^{\prime}, \mathrm{u}, \mathrm{p}, \mathrm{q}, \mathrm{s}\right)=\mathrm{c}^{2}\left(\Delta_{\mathrm{n}}^{\mathrm{m}}, \mathcal{M}^{\prime \prime} \circ \mathcal{M}^{\prime}, \mathrm{u}, \mathrm{p}, \mathrm{q}, \mathrm{s}\right)$.

Theorem 2.7 Let $\mathcal{M}^{\prime}=\left(M_{k, l}^{\prime}\right)$ and $\mathcal{M}^{\prime \prime}=\left(M_{k, 1}^{\prime \prime}\right)$ be two sequences of Orlicz functions, $\mathbf{q}, q_{1}$ and $\mathrm{q}_{2}$ be seminorms and $\mathrm{s}, \mathrm{s}_{1}$ and $\mathrm{s}_{2}$ be positive real numbers. Then

(1) $Z^{2}\left(\Delta_{n}^{m}, \mathcal{M}^{\prime}, u, p, q, s\right) \cap Z^{2}\left(\Delta_{n}^{m}, \mathcal{M}^{\prime \prime}, u, p, q, s\right) \subset Z^{2}\left(\Delta_{n}^{m}, \mathcal{M}^{\prime}+\mathcal{M}^{\prime \prime}, u, p, q, s\right)$,

(2) $Z^{2}\left(\Delta_{n}^{m}, \mathcal{M}, u, p, q_{1}, s\right) \cap Z^{2}\left(\Delta_{n}^{m}, \mathcal{M}, u, p, q_{2}, s\right) \subset Z^{2}\left(\Delta_{n}^{m}, \mathcal{M}, u, p, q_{1}+q_{2}, s\right)$,

(3) If $\mathrm{q}_{1}$ is stronger than $\mathrm{q}_{2}$, then $\mathrm{Z}^{2}\left(\Delta_{\mathrm{n}}^{\mathrm{m}}, \mathcal{M}, \mathrm{u}, \mathrm{p}, \mathrm{q}_{1}, \mathrm{~s}\right) \subset \mathrm{Z}^{2}\left(\Delta_{\mathrm{n}}^{\mathrm{m}}, \mathcal{M}, \mathrm{u}, \mathrm{p}, \mathrm{q}_{2}, \mathrm{~s}\right)$

(4) If $s_{1} \leq s_{2}$, then $Z^{2}\left(\Delta_{n}^{m}, \mathcal{M}, u, p, q, s_{1}\right) \subset Z^{2}\left(\Delta_{n}^{m}, \mathcal{M}, u, p, q, s_{2}\right)$,

where $\mathrm{Z}^{2}=\mathrm{c}^{2}, \mathrm{c}_{0}^{2}$ and $\mathrm{l}_{\infty}^{2}$.

Proof. (1) Let $x=\left(x_{k, l}\right) \in c^{2}\left(\Delta_{\mathfrak{n}}^{m}, \mathcal{M}^{\prime}, u, p, q, s\right) \cap c^{2}\left(\Delta^{m}, \mathcal{M}^{\prime \prime}, u, p, q, s\right)$. Then

$$
\begin{aligned}
& P-\lim _{k, l}(k l)^{-s} u_{k, l}\left[M_{k, l}^{\prime}\left(q\left(\frac{\Delta_{n}^{m} x_{k, l}-L}{\rho_{1}}\right)\right)\right]^{P_{k, l}}=0, \text { for some } \rho_{1}>0, \\
& P-\lim _{k, l}(k l)^{-s} u_{k, l}\left[M_{k, l}^{\prime \prime}\left(q\left(\frac{\Delta_{n}^{m} x_{k, l}-L}{\rho_{2}}\right)\right)\right]^{P_{k, l}}=0 \text {, for some } \rho_{2}>0 .
\end{aligned}
$$

Let $\rho=\max \left(\rho_{1}, \rho_{2}\right)$. The result follows from the following inequality

$$
\begin{aligned}
(\mathrm{kl})^{-s} & {\left[\left(\mathcal{M}^{\prime}+\mathcal{M}^{\prime \prime}\right)\left(\mathrm{q}\left(\frac{\Delta_{n}^{\mathrm{m}} x_{k, l}-\mathrm{L}}{\rho}\right)\right)\right]^{\mathrm{P}_{k, l}} } \\
& \leq \mathrm{K}\left\{(\mathrm{kl})^{-s} \mathfrak{u}_{k, l}\left[\mathcal{M}^{\prime}\left(\mathrm{q}\left(\frac{\Delta_{n}^{\mathrm{m}} x_{k, l}-\mathrm{L}}{\rho_{1}}\right)\right)\right]^{\mathrm{P}_{k, l}}+(\mathrm{kl})^{-s} \mathfrak{u}_{k, l}\left[\mathcal{M}^{\prime \prime}\left(\mathrm{q}\left(\frac{\Delta_{n}^{\mathrm{m}} x_{k, l}-\mathrm{L}}{\rho_{2}}\right)\right)\right]^{\mathrm{P}_{k, l}}\right\} .
\end{aligned}
$$

The proofs of (2), (3) and (4) follows by same pattern.

Theorem 2.8 For any sequence of orlicz functions, if $\mathrm{q}_{1} \equiv$ (equivalent to) $\mathrm{q}_{2}$, then $\mathrm{Z}^{2}\left(\Delta_{\mathrm{n}}^{\mathrm{m}}, \mathcal{M}, \mathrm{u}, \mathrm{p}, \mathrm{q}_{1}, \mathrm{~s}\right)=\mathrm{Z}^{2}\left(\Delta_{\mathrm{n}}^{\mathrm{m}}, \mathcal{M}, \mathrm{u}, \mathrm{p}, \mathrm{q}_{2}, \mathrm{~s}\right)$, where $\mathrm{Z}^{2}=\mathrm{c}^{2}, \mathrm{c}_{0}^{2}$ and $\mathrm{l}_{\infty}^{2}$.

Proof.It is easy to prove so we omit the details.

\section{Some generalized difference double sequence spaces over n-normed spaces}

The concept of 2-normed spaces was initially developed by Gähler[8] in the mid of 1960's, while that of $n$-normed spaces one can see in Misiak[16]. Since then, many others have studied this concept and obtained various results, see Gunawan ([9],[10]), Gunawan and Mashadi [11] and many others. 
Let $n \in \mathbb{N}$ and $X$ be a linear space over the field $\mathbb{K}$, where $\mathbb{K}$ is the field of real or complex numbers of dimension $d$, where $d \geq n \geq 2$. A real valued function $\|\cdot, \cdots, \cdot\|$ on $X^{n}$ satisfying the following four conditions:

(1) $\left\|x_{1}, x_{2}, \cdots, x_{n}\right\|=0$ if and only if $x_{1}, x_{2}, \cdots, x_{n}$ are linearly dependent in $X$;

(2) $\left\|x_{1}, x_{2}, \cdots, x_{n}\right\|$ is invariant under permutation;

(3) $\left\|\alpha x_{1}, x_{2}, \cdots, x_{n}\right\|=|\alpha|\left\|x_{1}, x_{2}, \cdots, x_{n}\right\|$ for any $\alpha \in \mathbb{K}$, and

(4) $\left\|x+x^{\prime}, x_{2}, \cdots, x_{n}\right\| \leq\left\|x, x_{2}, \cdots, x_{n}\right\|+\left\|x^{\prime}, x_{2}, \cdots, x_{n}\right\|$

is called an $n$-norm on $X$ and the pair $(X,\|\cdot, \cdots, \cdot\|)$ is called a $n$-normed space over the field $\mathbb{K}$. For example, we may take $X=\mathbb{R}^{n}$ being equipped with the $n$-norm $\left\|x_{1}, x_{2}, \cdots, x_{n}\right\|_{E}=$ the volume of the $n$-dimensional parallelopiped spanned by the vectors $x_{1}, x_{2}, \cdots, x_{n}$ which may be given explicitly by the formula

$$
\left\|x_{1}, x_{2}, \cdots, x_{n}\right\|_{E}=\left|\operatorname{det}\left(x_{i j}\right)\right|,
$$

where $x_{i}=\left(x_{i 1}, x_{i 2}, \cdots, x_{i n}\right) \in \mathbb{R}^{n}$ for each $i=1,2, \cdots, n$ and $\|.\|_{E}$ denotes the Euclidean norm. Let $(X,\|, \cdots, \cdot\|)$ be an $n$-normed space of dimension $d \geq n \geq 2$ and $\left\{a_{1}, a_{2}, \cdots, a_{n}\right\}$ be linearly independent set in $\mathrm{X}$. Then the following function $\|, \cdots, \cdot\|_{\infty}$ on $X^{n-1}$ defined by

$$
\left\|x_{1}, x_{2}, \cdots, x_{n-1}\right\|_{\infty}=\max \left\{\left\|x_{1}, x_{2}, \cdots, x_{n-1}, a_{i}\right\|: i=1,2, \cdots, n\right\}
$$

defines an $(n-1)$-norm on $X$ with respect to $\left\{a_{1}, a_{2}, \cdots, a_{n}\right\}$.

A sequence $\left(x_{k}\right)$ in a $n$-normed space $(X,\|\cdot, \cdots, \cdot\|)$ is said to converge to some $L \in X$ if

$$
\lim _{k \rightarrow \infty}\left\|x_{k}-L, z_{1}, \cdots, z_{n-1}\right\|=0 \text { for every } z_{1}, \cdots, z_{\mathfrak{n}-1} \in X .
$$

A sequence $\left(x_{k}\right)$ in a $n$-normed space $(X,\|\cdot, \cdots, \cdot\|)$ is said to be Cauchy if

$$
\lim _{k, p \rightarrow \infty}\left\|x_{k}-x_{p}, z_{1}, \cdots, z_{n-1}\right\|=0 \text { for every } z_{1}, \cdots, z_{n-1} \in X .
$$

If every Cauchy sequence in $X$ converges to some $L \in X$, then $X$ is said to be complete with respect to the n-norm. Any complete $n$-normed space is said to be n-Banach space. For more details about sequence spaces see ([28], [29]) and references therein.

Let $\mathcal{M}=\left(M_{k, l}\right)$ be a sequence of Orlicz functions, $p=\left(p_{k, l}\right)$ be a bounded sequence of positive real numbers and $u=\left(u_{k, l}\right)$ be a sequence of positive reals such that $\mathfrak{u}_{k, l} \neq 0$ for all $k$, then we define the following sequences spaces in the present paper:

$$
\begin{aligned}
& c_{0}^{2}\left(\mathcal{M}, \Delta_{n}^{m}, p, u, s,\|\cdot, \cdots, \cdot\|\right)= \\
& \left\{x=\left(x_{k, l}\right) \in w^{2}: \lim _{k, l \rightarrow \infty}(k l)^{-s} u_{k, l}\left[M_{k, l}\left(\left\|\frac{\Delta_{n}^{m} x_{k, l}}{\rho}, z_{1}, \cdots, z_{n-1}\right\|\right)\right]^{p_{k, l}}=0,\right.
\end{aligned}
$$


for some $\rho>0$ and $s \geq 0\}$,

$c^{2}\left(\mathcal{M}, \Delta_{\mathfrak{n}}^{m}, p, u, s,\|\cdot, \cdots, \cdot\|\right)=$

$$
\left\{x=\left(x_{k, l}\right) \in w^{2}: \lim _{k, l \rightarrow \infty}(k l)^{-s} u_{k, l}\left[M_{k, l}\left(\left\|\frac{\Delta_{n}^{m} x_{k, l}-L}{\rho}, z_{1}, \cdots, z_{n-1}\right\|\right)\right]^{p_{k, l}}=0\right.
$$

for some $\rho>0, L$ and $s \geq 0\}$,

and

$l_{\infty}^{2}\left(\mathcal{M}, \Delta_{n}^{m}, p, u, s,\|\cdot, \cdots, \cdot\|\right)=$

$$
\left\{x=\left(x_{k, l}\right) \in w^{2}: \sup _{k, l \geq 1}(k l)^{-s} u_{k, l}\left[M_{k, l}\left(\left\|\frac{\Delta_{n}^{m} x_{k, l}}{\rho}, z_{1}, \cdots, z_{n-1}\right\|\right)\right]^{p_{k, l}}<\infty\right.
$$

for some $\rho>0$ and $s \geq 0\}$.

In this section of the present paper we shall study the topological properties and some interesting inclusion relation between the $\operatorname{spaces}^{2}\left(\mathcal{M}, \Delta_{\mathfrak{n}}^{\mathrm{m}}, \mathrm{p}, \mathrm{u}, \mathrm{s},\|\cdot, \cdots, \cdot\|\right), \mathrm{c}_{0}^{2}\left(\mathcal{M}, \Delta_{\mathfrak{n}}^{\mathrm{m}}, \mathrm{p}, \mathrm{u}, \mathrm{s},\|\cdot, \cdots, \cdot\|\right)$ and $l_{\infty}^{2}\left(\mathcal{M}, \Delta_{n}^{m}, p, u, s,\|\cdot, \cdots, \cdot\|\right)$.

Theorem 3.1 Let $\mathcal{M}=\left(M_{k, l}\right)$ be a sequence of Orlicz functions, $p=\left(p_{k, l}\right)$ be a bounded sequence of positive real numbers and $\mathfrak{u}=\left(\mathfrak{u}_{k, l}\right)$ be a sequence of strictly positive real numbers, then the spaces $\mathrm{c}_{0}^{2}\left(\mathcal{M}, \Delta_{\mathrm{n}}^{\mathrm{m}}, \mathrm{p}, \mathrm{u}, \mathrm{s},\|\cdot, \cdots, \cdot\|\right), \mathrm{c}^{2}\left(\mathcal{M}, \Delta_{\mathfrak{n}}^{\mathrm{m}}, \mathrm{p}, \mathrm{u}, \mathrm{s},\|\cdot, \cdots, \cdot\|\right)$ and $\mathrm{l}_{\infty}^{2}\left(\mathcal{M}, \Delta_{\mathrm{n}}^{\mathrm{m}}, \mathrm{p}, \mathrm{u}, \mathrm{s},\|\cdot, \cdots, \cdot \cdot\|\right)$ are linear spaces.

Proof. Let $x=\left(x_{k, l}\right), y=\left(y_{k, l}\right) \in c_{0}^{2}\left(\mathcal{M}, \Delta_{n}^{m}, p, u, s,\|\cdot, \cdots, \cdot\|\right)$ and $\alpha, \beta \in \mathbb{C}$. Then there exist positive number $\rho_{1}$ and $\rho_{2}$ such that

$$
\lim _{k, l \rightarrow \infty}(k l)^{-s} u_{k, l}\left[M_{k, l}\left(\left\|\frac{\Delta_{n}^{m} x_{k, l}}{\rho_{1}}, z_{1}, \cdots, z_{n-1}\right\|\right)\right]^{p_{k, l}}=0, \text { for some } \rho_{1}>0
$$

and

$$
\lim _{k, l \rightarrow \infty}(k l)^{-s} u_{k, l}\left[M_{k, l}\left(\left\|\frac{\Delta_{n}^{m} y_{k, l}}{\rho_{2}}, z_{1}, \cdots, z_{n-1}\right\|\right)\right]^{p_{k, l}}=0, \text { for some } \rho_{2}>0
$$

Let $\rho_{3}=\max \left(2|\alpha| \rho_{1}, 2|\beta| \rho_{2}\right)$. Since $\mathcal{M}=\left(M_{k, l}\right)$ is non-decreasing convex function and so by using inequality (1.1), we have 


$$
\begin{aligned}
\lim _{k, l \rightarrow \infty} & (k l)^{-s} u_{k, l}\left[M_{k, l}\left(\left\|\frac{\Delta_{n}^{m}\left(\alpha x_{k, l}+\beta y_{k, l}\right) \mid}{\rho_{3}}, z_{1}, \cdots, z_{n-1}\right\|\right)\right]^{p_{k, l}} \\
& =\lim _{k, l \rightarrow \infty}(k l)^{-s} u_{k, l}\left[M_{k, l}\left(\left\|\frac{\alpha \Delta_{n}^{m} x_{k, l}}{\rho_{3}}, z_{1}, \cdots, z_{n-1}\right\|+\left\|\frac{\beta \Delta_{n}^{m} y_{k, l}}{\rho_{3}}, z_{1}, \cdots, z_{n-1}\right\|\right)\right]^{p_{k, l}} \\
& \leq K \lim _{k, l \rightarrow \infty} \frac{1}{2^{p_{k, l}}}(k l)^{-s} u_{k, l}\left[M_{k, l}\left(\left\|\frac{\Delta_{n}^{m} x_{k, l}}{\rho_{1}}, z_{1}, \cdots, z_{n-1}\right\|\right)\right]^{p_{k, l}} \\
& +K \lim _{k, l \rightarrow \infty} \frac{1}{2^{p_{k, l}}}(k l)^{-s} u_{k, l}\left[M_{k, l}\left(\left\|\frac{\Delta_{n}^{m} y_{k, l}}{\rho_{2}}, z_{1}, \cdots, z_{n-1}\right\|\right)\right]^{p_{k, l}} \\
& \leq K \lim _{k, l \rightarrow \infty}(k l)^{-s} u_{k, l}\left[M_{k, l}\left(\left\|\frac{\Delta_{n}^{m} x_{k, l}}{\rho_{1}}, z_{1}, \cdots, z_{n-1}\right\|\right)\right]^{p_{k, l}} \\
& +K \lim _{k, l \rightarrow \infty}(k l)^{-s} u_{k, l}\left[M_{k, l}\left(\left\|\frac{\Delta_{n}^{m} y_{k, l}}{\rho_{2}}, z_{1}, \cdots, z_{n-1}\right\|\right)\right]^{p_{k, l}} \\
& =0 .
\end{aligned}
$$

So, $\alpha x+\beta y \in c_{0}^{2}\left(\mathcal{M}, \Delta_{m}^{n}, p, u, s,\|\cdot, \cdots, \cdot\|\right)$. Hence $c_{0}^{2}\left(\mathcal{M}, \Delta_{m}^{n}, p, u, s,\|\cdot, \cdots, \cdot\|\right)$ is a linear space. Similarly, we can prove that $c^{2}\left(\mathcal{M}, \Delta_{\mathfrak{n}}^{\mathrm{m}}, \mathrm{p}, \mathrm{u}, \mathrm{s},\|\cdot, \cdots, \cdot\|\right)$ and $\mathrm{l}_{\infty}^{2}\left(\mathcal{M}, \Delta_{\mathfrak{n}}^{\mathrm{m}}, \mathrm{p}, \mathrm{u}, \mathrm{s},\|\cdot, \cdots, \cdot\|\right)$ are linear spaces.

Theorem 3.2 Let $\mathcal{M}=\left(M_{k, l}\right)$ be a sequence of Orlicz functions, $p=\left(p_{k, l}\right)$ be a bounded sequence of positive real numbers and $\mathrm{u}=\left(\mathfrak{u}_{\mathrm{k}, \mathrm{l}}\right)$ be a sequence of strictly positive real numbers. For $\mathrm{Z}^{2}=\mathrm{l}_{\infty}^{2}, \mathrm{c}^{2}$ and $\mathrm{c}_{\mathrm{o}}^{2}$, the spaces $\mathrm{Z}^{2}\left(\mathcal{M}, \Delta_{\mathfrak{n}}^{\mathrm{m}}, \mathrm{p}, \mathrm{u}, \mathrm{s},\|\cdot, \cdots, \cdot\|\right)$ are paranormed spaces, paranormed by

$g(x)=\sum_{k, l=1}^{n m}\left\|x_{k, l}, z_{1}, \cdots, z_{n-1}\right\|+\inf \left\{\rho^{\frac{p_{k, l}}{H}}: \sup _{k, l}(k l)^{-s} u_{k, l} M_{k, l}\left(\left\|\frac{\Delta_{n}^{m} x_{k, l}}{\rho}, z_{1}, \cdots, z_{n-1}\right\|\right) \leq 1\right\}$ where $\mathrm{H}=\max \left(1, \sup _{\mathrm{k}, \mathrm{l}} \mathrm{p}_{\mathrm{k}, \mathrm{l}}\right)$.

Proof. Clearly $g(-x)=g(x), g(0)=0$. Let $\left(x_{k}\right)$ and $\left(y_{k}\right)$ be any two sequences belong to any one of the spaces $Z^{2}\left(\mathcal{M}, \Delta_{n}^{m}, p, u, s,\|\cdot, \cdots, \cdot\|\right)$, for $Z^{2}=c_{0}^{2}, c^{2}$ and $l_{\infty}^{2}$. Then, we get $\rho_{1}, \rho_{2}>0$ such that

$$
\sup _{k, l}(k l)^{-s} \mathfrak{u}_{k, l}\left[M_{k, l}\left(\left\|\frac{\Delta_{n}^{m} x_{k, l}}{\rho_{1}}, z_{1}, \cdots, z_{n-1}\right\|\right)\right] \leq 1
$$

and

$$
\sup _{k, l}(k l)^{-s} \mathfrak{u}_{k, l}\left[M_{k, l}\left(\left\|\frac{\Delta_{n}^{m} y_{k, l}}{\rho_{2}}, z_{1}, \cdots, z_{n-1}\right\|\right)\right] \leq 1 .
$$

Let $\rho=\rho_{1}+\rho_{2}$. Then by convexity of $\mathcal{M}=\left(M_{k, l}\right)$, we have

$$
\begin{aligned}
\sup _{k, l}(k l)^{-s} \mathfrak{u}_{k, l}\left[M_{k, l}\left(\left\|\frac{\Delta_{n}^{\mathrm{m}}\left(x_{k, l}+y_{k, l}\right)}{\rho}, z_{1}, \cdots, z_{n-1}\right\|\right)\right] \\
\leq\left(\frac{\rho_{1}}{\rho_{1}+\rho_{2}}\right) \sup _{k, l}(k l)^{-s} \mathfrak{u}_{k, l}\left[M_{k, l}\left(\left\|\frac{\Delta_{n}^{m} x_{k, l}}{\rho_{1}}, z_{1}, \cdots, z_{n-1}\right\|\right)\right] \\
+\left(\frac{\rho_{2}}{\rho_{1}+\rho_{2}}\right) \sup _{k, l}(k l)^{-s} u_{k, l}\left[M_{k, l}\left(\left\|\frac{\Delta_{n}^{m} y_{k, l}}{\rho_{2}}, z_{1}, \cdots, z_{n-1}\right\|\right)\right] \\
\leq 1 .
\end{aligned}
$$


Hence we have,

$$
\begin{aligned}
g(x+y) & =\sum_{k, l=1}^{m n}\left\|\left(x_{k, l}+y_{k, l}\right), z_{1}, \cdots, z_{n-1}\right\| \\
& +\inf \left\{\rho^{\frac{p_{k}, l}{H}}: \sup _{k, l}(k l)^{-s} u_{k, l}\left[M_{k, l}\left(\left\|\frac{\Delta_{n}^{m}\left(x_{k, l}+y_{k, l}\right)}{\rho}, z_{1}, \cdots, z_{n-1}\right\|\right)\right] \leq 1\right\} \\
& \leq \sum_{k, l=1}^{m n}\left\|x_{k, l}, z_{1}, \cdots, z_{n-1}\right\| \\
& +\inf \left\{\rho_{1}^{\frac{p_{k}, l}{H}}: \sup _{k, l}(k l)^{-s} u_{k, l}\left[M_{k, l}\left(\left\|\frac{\Delta_{n}^{m} x_{k, l}}{\rho_{1}}, z_{1}, \cdots, z_{n-1}\right\|\right)\right] \leq 1\right\} \\
& +\sum_{k, l=1}^{m n}\left\|y_{k, l}, z_{1}, \cdots, z_{n-1}\right\| \\
& +\inf \left\{\rho_{2}^{\frac{p_{k, l}}{H}}: \sup _{k, l}(k l)^{-s} u_{k, l}\left[M_{k, l}\left(\left\|\frac{\Delta_{n}^{m} y_{k, l}}{\rho_{2}}, z_{1}, \cdots, z_{n-1}\right\|\right)\right] \leq 1\right\} .
\end{aligned}
$$

This implies that

$$
g(x+y) \leq g(x)+g(y) .
$$

The continuity of the scalar multiplication follows from the following inequality

$$
\begin{aligned}
g(\mu x) & =\sum_{k, l=1}^{m n}\left\|\mu x_{k, l}, z_{1}, \cdots, z_{n-1}\right\| \\
& +\inf \left\{\rho^{\frac{p_{k}, l}{H}}: \sup _{k, l}(k l)^{-s} u_{k, l}\left[M_{k, l}\left(\left\|\frac{\Delta_{n}^{m} \mu x_{k, l}}{\rho}, z_{1}, \cdots, z_{n-1}\right\|\right)\right] \leq 1\right\} \\
& =|\mu| \sum_{k, l=1}^{m n}\left\|x_{k, l}, z_{1}, \cdots, z_{n-1}\right\| \\
& +\inf \left\{(t|\mu|)^{\frac{p_{k, l}}{H}}: \sup _{k, l}(k l)^{-s} u_{k, l}\left[M_{k, l}\left(\left\|\frac{\Delta_{n}^{m} x_{k, l}}{t}, z_{1}, \cdots, z_{\mathfrak{n}-1}\right\|\right)\right] \leq 1\right\},
\end{aligned}
$$

where $t=\frac{\rho}{|\mu|}$. Hence the space $Z^{2}\left(\mathcal{M}, \Delta_{\mathfrak{n}}^{m}, p, u, s,\|\cdot, \cdots, \cdot\|\right)$, for $Z^{2}=c_{0}^{2}, c^{2}$ and $l_{\infty}^{2}$ is a paranormed space, paranormed by $g$.

Theorem 3.3 Let $\mathcal{M}=\left(M_{k, l}\right)$ be a sequence of Orlicz functions, $p=\left(p_{k, l}\right)$ be a bounded sequence of positive real numbers and $\mathfrak{u}=\left(\mathfrak{u}_{k, l}\right)$ be a sequence of strictly positive real numbers. For $\mathrm{Z}^{2}=\mathrm{l}_{\infty}^{2}, \mathrm{c}^{2}$ and $\mathrm{c}_{0}^{2}$, the spaces $\mathrm{Z}^{2}\left(\mathcal{M}, \Delta_{\mathrm{n}}^{\mathrm{m}}, \mathrm{p}, \mathrm{u}, \mathrm{s},\|\cdot, \cdots, \cdot\|\right)$ are complete paranormed spaces, paranormed by

$g(x)=\sum_{k, l=1}^{n m}\left\|x_{k, l}, z_{1}, \cdots, z_{n-1}\right\|+\inf \left\{\rho^{\frac{p_{k, l}}{H}}: \sup _{k, l}(k l)^{-s} u_{k, l} M_{k, l}\left(\left\|\frac{\Delta_{n}^{m} x_{k, l}}{\rho}, z_{1}, \cdots, z_{n-1}\right\|\right) \leq 1\right\}$, where $\mathrm{H}=\max \left(1, \sup _{\mathrm{k}, \mathrm{l}} \mathrm{p}_{\mathrm{k}, \mathrm{l}}\right)$.

Proof. We prove the result for the space $l_{\infty}^{2}\left(\mathcal{M}, \Delta_{\mathfrak{n}}^{m}, p, u, s,\|\cdot, \cdots, \cdot\|\right)$. Let $\left(x^{i}\right)$ be any Cauchy 
sequence in $l_{\infty}^{2}\left(\mathcal{M}, \Delta_{n}^{m}, p, u, s,\|\cdot, \cdots, \cdot\|\right)$. Let $\epsilon>0$ be given and for $t>0$, choose $x_{0}$ be fixed such that $u_{k, l} M_{k, l}\left(\frac{t x_{0}}{2}\right) \geq 1$, then there exists a positive integer $n_{0} \in \mathbb{N}$ such that $g\left(x_{k, l}^{i}-x_{k, l}^{j}\right)<\frac{\epsilon}{x_{0} t}$, for all $i, j \geq n_{0}$. Using the definition of paranorm, we get

$$
\begin{aligned}
& \sum_{k, l=1}^{m n}\left\|\left(x_{k, l}^{i}-x_{k, l}^{j}\right), z_{1}, \cdots, z_{n-1}\right\|+\inf \left\{\rho^{\frac{p_{k, l} l}{H}}: \sup _{k, l}(k l)^{-s} u_{k, l} M_{k, l}\left(\left\|\frac{\Delta_{n}^{m}\left(x_{k, l}^{i}-x_{k, l}^{j}\right)}{\rho}, z_{1}, \cdots, z_{n-1}\right\|\right)\right\} \\
&<\frac{\epsilon}{x_{0} t}, \text { for all } i, j \geq n_{0}
\end{aligned}
$$

Hence we have,

$$
\left.\sum_{k, l=1}^{m n} \|\left(x_{k, l}^{i}\right)-x_{k, l}^{j}\right), z_{1}, \cdots, z_{n-1} \|<\epsilon, \text { for all } i, j \geq n_{o}
$$

This implies that

$$
\left\|\left(x_{k, l}^{i}-x_{k, l}^{j}\right), z_{1}, \cdots, z_{n-1}\right\|<\epsilon \text {, for all } i, j \geq n_{0} \text { and } 1 \leq k \leq n m .
$$

Thus $\left(x_{k, l}^{i}\right)$ is a Cauchy sequence in $\mathbb{C}$ for $k, l=1,2, \ldots ., n m$. Hence $\left(x_{k, l}^{i}\right)$ is convergent in $\mathbb{C}$ for $k, l=1,2, \ldots, n m$. Let $\lim _{i \rightarrow \infty} x_{k, l}^{i}=x_{k, l}$, say for $k, l=1,2, \ldots, n m$.

Again from equation (3.1) we have,

$$
\inf \left\{\rho^{\frac{p_{k, l}}{H}}: \sup _{k, l}(k l)^{-s} u_{k, l} M_{k, l}\left(\left\|\frac{\Delta_{n}^{m}\left(x_{k, l}^{i}-x_{k, l}^{j}\right)}{\rho}, z_{1}, \cdots, z_{n-1}\right\|\right) \leq 1\right\}<\epsilon, \text { for all } i, j \geq n_{0} .
$$

Hence we get

$$
\sup _{k, l}(k l)^{-s} u_{k, l} M_{k, l}\left(\left\|\frac{\Delta_{n}^{m}\left(x_{k, l}^{i}-x_{k, l}^{j}\right)}{g\left(x^{i}-x^{j}\right)}, z_{1}, \cdots, z_{n-1}\right\|\right) \leq 1, \text { for all } i, j \geq n_{0} .
$$

It follows that $(k l)^{-s} \mathfrak{u}_{k, l} M_{k, l}\left(\left\|\frac{\Delta_{n}^{m}\left(x_{k, l}^{i}-x_{k, l}^{j}\right)}{g\left(x^{i}-x^{j}\right)}, z_{1}, \cdots, z_{n-1}\right\|\right) \leq 1$, for each $k, l \geq 1$ and for all $i, j \geq n_{0}$.

For $\mathrm{t}>0$ with $(k l)^{-s} \mathfrak{u}_{k, l} M_{k, l}\left(\frac{\mathrm{t} x_{0}}{2}\right) \geq 1$, we have

$$
(k l)^{-s} \mathfrak{u}_{k, l} M_{k, l}\left(\left\|\frac{\Delta_{n}^{m}\left(x_{k, l}^{i}-x_{k, l}^{j}\right)}{g\left(x^{i}-x^{j}\right)}, z_{1}, \cdots, z_{n-1}\right\|\right) \leq(k l)^{-s} u_{k, l} M_{k, l}\left(\frac{t x_{0}}{2}\right) .
$$

This implies that

$$
\left\|\left(\Delta_{n}^{m} x_{k, l}^{i}-\Delta_{n}^{m} x_{k, l}^{j}\right), z_{1}, \cdots, z_{n-1}\right\|<\frac{t x_{0}}{2} \frac{\epsilon}{t x_{0}}=\frac{\epsilon}{2} .
$$

Hence $\left(\Delta_{n}^{m} x_{k, l}^{i}\right)$ is a Cauchy sequence in $\mathbb{C}$ for all $k, l \in \mathbb{N}$. This implies that $\left(\Delta_{n}^{m} x_{k, l}^{i}\right)$ is convergent in $\mathbb{C}$ for all $k, l \in \mathbb{N}$. Let $\lim _{i \rightarrow \infty} \Delta_{n}^{m} x_{k, l}^{i}=y_{k, l}$ for each $k, l \in \mathbb{N}$. Let $k, l=1$, then we have

$$
\lim _{i \rightarrow \infty} \Delta_{n}^{m} x_{1,1}^{i}=\lim _{i \rightarrow \infty} \sum_{v=0}^{n}(-1)^{v}\left(\begin{array}{c}
m \\
v
\end{array}\right) x_{1+n v, m v+1}^{i}=y_{1} .
$$


We have by equation (3.2) and equation (3.3) $\lim _{i \rightarrow \infty} x_{m n+1}^{i}=x_{m n+1}$, exists. Proceeding in this way inductively, we have $\lim _{i \rightarrow \infty} x_{k, l}^{i}=x_{k, l}$ exists for each $k, l \in \mathbb{N}$. Now we have for all $i, j \geq n_{0}$,

$$
\begin{aligned}
& \sum_{k, l=1}^{m n}\left\|\left(x_{k, l}^{i}-x_{k, l}^{j}\right), z_{1}, \cdots, z_{n-1}\right\| \\
& \quad+\inf \left\{\rho^{\frac{p_{k, l}}{H}}: \sup _{k, l}(k l)^{-s} u_{k, l} M_{k, l}\left(\left\|\frac{\Delta_{n}^{m}\left(x_{k, l}^{i}-x_{k, l}^{j}\right)}{\rho}, z_{1}, \cdots, z_{n-1}\right\|\right) \leq 1\right\}<\epsilon .
\end{aligned}
$$

This implies that

$$
\begin{aligned}
& \lim _{j \rightarrow \infty}\left\{\sum_{k, l=1}^{m n}\left\|\left(x_{k, l}^{i}-x_{k, l}^{j}\right), z_{1}, \cdots, z_{n-1}\right\|\right. \\
& \left.\quad+\inf \left\{\rho^{\frac{p_{k}, l}{H}}: \sup _{k, l}(k l)^{-s} u_{k, l} M_{k, l}\left(\left\|\frac{\Delta_{n}^{m}\left(x_{k, l}^{i}-x_{k, l}^{j}\right)}{\rho}, z_{1}, \cdots, z_{n-1}\right\|\right) \leq 1\right\}\right\}<\epsilon,
\end{aligned}
$$

for all $i \geq n_{0}$. Using the continuity of $\left(M_{k, l}\right)$, we have

$$
\begin{aligned}
& \sum_{k, l=1}^{m n}\left\|\left(x_{k, l}^{i}-x_{k, l}\right), z_{1}, \cdots, z_{n-1}\right\| \\
& \quad+\inf \left\{\rho^{\frac{p_{k}, l}{H}}: \sup _{k, l}(k l)^{-s} u_{k, l} M_{k, l}\left(\left\|\frac{\left(\Delta_{n}^{m} x_{k, l}^{i}-\Delta_{n}^{m} x_{k, l}\right)}{\rho}, z_{1}, \cdots, z_{n-1}\right\|\right) \leq 1\right\}<\epsilon,
\end{aligned}
$$

for all $i \geq n_{0}$. It follows that $\left(x^{i}-x\right) \in l_{\infty}^{2}\left(\mathcal{M}, \Delta_{n}^{m}, p, u, s,\|\cdot, \cdots, \cdot\|\right)$. Since $x^{i} \in$ $l_{\infty}^{2}\left(\mathcal{M}, \Delta_{m}^{n}, p, u, s,\|\cdot, \cdots, \cdot\|\right)$ and $l_{\infty}^{2}\left(\mathcal{M}, \Delta_{m}^{n}, p, u, s,\|\cdot, \cdots, \cdot\|\right)$ is a linear space, so we have $x=$ $x^{i}-\left(x^{i}-x\right) \in l_{\infty}^{2}\left(\mathcal{M}, \Delta_{m}^{n}, p, u, s,\|\cdot, \cdots, \cdot\|\right)$. This completes the proof. Similarly, we can prove that $\mathrm{c}^{2}\left(\mathcal{M}, \Delta_{\mathrm{m}}^{\mathrm{n}}, \mathrm{p}, \mathrm{u},\|\cdot, \cdots, \cdot\|\right)$ and $\mathrm{c}_{0}^{2}\left(\mathcal{M}, \Delta_{\mathrm{m}}^{\mathrm{n}}, \mathrm{p}, \mathrm{u},\|\cdot, \cdots, \cdot\|\right)$ are complete paranormed spaces in view of the above proof.

Theorem 3.4 If $0<p_{k, l} \leq q_{k, l}<\infty$ for each $k, l$, then $Z^{2}\left(\mathcal{M}, \Delta_{\mathfrak{n}}^{m}, p, u, s,\|\cdot, \cdots, \cdot\|\right) \subseteq$ $\mathrm{Z}^{2}\left(\mathcal{M}, \Delta_{\mathfrak{n}}^{\mathrm{m}}, \mathrm{q}, \mathrm{u}, \mathrm{s},\|\cdot, \cdots, \cdot\|\right)$, for $\mathrm{Z}^{2}=\mathrm{c}_{0}^{2}$ and $\mathrm{c}^{2}$.

Proof. Let $x=\left(x_{k, l}\right) \in c^{2}\left(\mathcal{M}, \Delta_{\mathfrak{n}}^{m}, p, u, s,\|\cdot, \cdots, \cdot\|\right)$. Then there exists some $\rho>0$ and $L \in X$ such that

$$
\lim _{k, l \rightarrow \infty}(k l)^{-s} u_{k, l}\left(M_{k, l}\left(\left\|\frac{\Delta_{n}^{m} x_{k, l}-L}{\rho}, z_{1}, \cdots, z_{n-1}\right\|\right)\right)^{p_{k, l}}=0
$$

This implies that $(k l)^{-s} \mathfrak{u}_{k, l} M_{k, l}\left(\left\|\frac{\Delta_{n}^{m} \chi_{k, l}-L}{\rho}, z_{1}, \cdots, z_{n-1}\right\|\right)<\epsilon \quad(0<\epsilon<1)$ for sufficiently large $k, l$. Hence we get

$$
\begin{aligned}
\lim _{k, l \rightarrow \infty}(k l)^{-s} u_{k, l} & \left(M_{k, l}\left(\left\|\frac{\Delta_{n}^{m} x_{k, l}-L}{\rho}, z_{1}, \cdots, z_{n-1}\right\|\right)\right)^{q_{k, l}} \\
\leq & \lim _{k, l \rightarrow \infty}(k l)^{-s} u_{k, l}\left(M_{k, l}\left(\left\|\frac{\Delta_{n}^{m} x_{k, l}-L}{\rho}, z_{1}, \cdots, z_{n-1}\right\|\right)\right)^{p_{k, l}}=0 .
\end{aligned}
$$

This implies that $x=\left(x_{k, l}\right) \in c^{2}\left(\mathcal{M}, \Delta_{\mathfrak{n}}^{m}, q, u, s,\|\cdot, \cdots, \cdot\|\right)$. This completes the proof. Similarly, we can prove for the case $Z^{2}=c_{0}^{2}$. 
Theorem 3.5 If $\mathcal{M}^{\prime}=\left(M_{k, l}^{\prime}\right)$ and $\mathcal{M}^{\prime \prime}=\left(M_{k, l}^{\prime \prime}\right)$ be a sequence of Orlicz functions. Then (i) $Z^{2}\left(\mathcal{M}^{\prime}, \Delta_{\mathfrak{n}}^{m}, p, u, s,\|\cdot, \cdots, \cdot\|\right) \subseteq Z^{2}\left(\mathcal{M}^{\prime \prime} \circ \mathcal{M}^{\prime}, \Delta_{\mathfrak{n}}^{m}, p, u, s,\|\cdot, \cdots, \cdot\|\right)$,

$$
\begin{gathered}
Z^{2}\left(\mathcal{M}^{\prime}, \Delta_{\mathrm{n}}^{\mathrm{m}}, p, u, s,\|\cdot, \cdots, \cdot\|\right) \cap \mathrm{Z}^{2}\left(\mathcal{M}^{\prime \prime}, \Delta_{\mathrm{n}}^{\mathrm{m}}, \mathrm{p}, \mathrm{u}, \mathrm{s},\|\cdot, \cdots, \cdot\|\right) \\
\subseteq \mathrm{Z}^{2}\left(\mathcal{M}^{\prime}+\mathcal{M}^{\prime \prime}, \Delta_{\mathrm{n}}^{\mathrm{m}}, \mathrm{p}, \mathrm{u}, \mathrm{s},\|\cdot, \cdots, \cdot\|\right),
\end{gathered}
$$

for $\mathrm{Z}^{2}=\mathrm{l}_{\infty}^{2}, \mathrm{c}^{2}$ and $\mathrm{c}_{0}^{2}$

Proof. (i) We prove this part for $Z^{2}=l_{\infty}^{2}$ and the rest of the cases will follow similarly. Let $\left(x_{k, l}\right) \in l_{\infty}^{2}\left(\mathcal{M}^{\prime}, \Delta_{n}^{m}, p, u, s,\|\cdot, \cdots, \cdot\|\right)$, then there exists $0<\mathrm{U}<\infty$ such that

$$
(k l)^{-s} \mathfrak{u}_{k, l}\left(M_{k, l}^{\prime}\left(\left\|\frac{\Delta_{n}^{m} x_{k, l}}{\rho}, z_{1}, \cdots, z_{n-1}\right\|\right)\right)^{p_{k, l}} \leq \mathrm{U} \text {, for all } k, l \in \mathbb{N} .
$$

Let $\boldsymbol{y}_{k, l}=(k l)^{-s} \mathfrak{u}_{k, l} M_{k, l}^{\prime}\left(\left\|\frac{\Delta_{n}^{m} x_{k, l}}{\rho}, z_{1}, \cdots, z_{n-1}\right\|\right)$. Then $y_{k, l} \leq u^{\frac{1}{p_{k, l}}} \leq V$, say for all $k, l \in \mathbb{N}$. Hence we have

$$
\left(\left(M_{k, l}^{\prime \prime} \circ M_{k, l}^{\prime}\right)\left(\left\|\frac{\Delta_{n}^{m} x_{k, l}}{\rho}, z_{1}, \cdots, z_{n-1}\right\|\right)\right)^{p_{k, l}}=\left(M_{k, l}^{\prime \prime}\left(y_{k, l}\right)\right)^{p_{k, l}} \leq\left(M_{k, l}^{\prime \prime}(V)\right)^{p_{k, l}}<\infty,
$$

for all $k, l \in \mathbb{N}$.

Hence $\sup _{k, l} u_{k, l}\left(\left(M_{k, l}^{\prime \prime} \circ M_{k, l}^{\prime}\right)\left(\left\|\frac{\Delta_{n}^{m} x_{k, l}}{\rho}, z_{1}, \cdots, z_{n-1}\right\|\right)\right)^{p_{k, l}}<\infty$. Thus $x=\left(x_{k, l}\right) \in l_{\infty}^{2}\left(\mathcal{M}^{\prime \prime} \circ\right.$ $\left.\mathcal{M}^{\prime}, \Delta_{\mathrm{n}}^{\mathrm{m}}, \mathrm{p}, \mathrm{u}, \mathrm{s},\|\cdot, \cdots, \cdot\|\right)$.

(ii) We prove the result for the case $Z^{2}=c^{2}$ and the rest of the cases will follow similarly. Let $x=\left(x_{k, l}\right) \in c^{2}\left(\mathcal{M}^{\prime}, \Delta_{n}^{m}, p, u, s,\|\cdot, \cdots, \cdot\|\right) \cap c^{2}\left(\mathcal{M}^{\prime \prime}, \Delta_{n}^{m}, p, u, s,\|\cdot, \cdots, \cdot\|\right)$, then there exist some $\rho_{1}, \rho_{2}>0$ and $L \in X$ such that

$$
\lim _{k \rightarrow \infty}(k l)^{-s} u_{k, l}\left(M_{k, l}^{\prime}\left(\left\|\frac{\Delta_{n}^{m} \chi_{k, l}-L}{\rho_{1}}, z_{1}, \cdots, z_{n-1}\right\|\right)\right)^{p_{k, l}}=0
$$

and

$$
\lim _{k \rightarrow \infty}(k l)^{-s} u_{k, l}\left(M_{k, l}^{\prime \prime}\left(\left\|\frac{\Delta_{n}^{m} x_{k, l}-L}{\rho_{2}}, z_{1}, \cdots, z_{n-1}\right\|\right)\right)^{p_{k, l}}=0 .
$$

Let $\rho=\rho_{1}+\rho_{2}$. Then we have

$$
\begin{aligned}
(\mathrm{kl})^{-s} \mathfrak{u}_{k, l}\left(\left(M_{k, l}^{\prime}+\right.\right. & \left.\left.M_{k, l}^{\prime \prime}\right)\left(\left\|\frac{\Delta_{n}^{m} x_{k, l}-\mathrm{L}}{\rho}, z_{1}, \cdots, z_{\mathfrak{n}-1}\right\|\right)\right)^{p_{k, l}} \\
\leq & K\left[\left(\frac{\rho_{1}}{\rho_{1}+\rho_{2}}\right)(k l)^{-s} u_{k, l} M_{k, l}^{\prime}\left(\left\|\frac{\Delta_{n}^{m} x_{k, l}-L}{\rho_{1}}, z_{1}, \cdots, z_{n-1}\right\|\right)\right]^{p_{k, l}} \\
& +K\left[\left(\frac{\rho_{2}}{\rho_{1}+\rho_{2}}\right)(k l)^{-s} u_{k, l} M_{k, l}^{\prime \prime}\left(\left\|\frac{\Delta_{n}^{m} x_{k, l}-L}{\rho_{2}}, z_{1}, \cdots, z_{n-1}\right\|\right)\right]^{p_{k, l}} .
\end{aligned}
$$

This implies that

$$
\lim _{k \rightarrow \infty}(k l)^{-s} u_{k, l}\left(\left(M_{k, l}^{\prime}+M_{k, l}^{\prime \prime}\right)\left(\left\|\frac{\Delta_{n}^{m} x_{k, l}-L}{\rho}, z_{1}, \cdots, z_{n-1}\right\|\right)\right)^{p_{k, l}}=0 .
$$


Thus $x=\left(x_{k, l}\right) \in c^{2}\left(\mathcal{M}^{\prime}+\mathcal{M}^{\prime \prime}, \Delta_{m}^{m}, p, u, s,\|\cdot, \cdots, \cdot\|\right)$. This completes the proof.

Theorem 3.6 Let $\mathcal{M}=\left(\mathrm{M}_{\mathrm{k}, \mathrm{l}}\right)$ be a sequence of Orlicz functions, $\mathrm{p}=\left(\mathrm{p}_{\mathrm{k}, \mathrm{l}}\right)$ be a bounded sequence of positive real numbers and $\mathrm{u}=\left(\mathrm{u}_{\mathrm{k}, \mathrm{l}}\right)$ be a sequence of strictly positive real numbers, then $\mathrm{Z}^{2}\left(\mathcal{M}, \Delta_{\mathrm{n}}^{\mathrm{m}-1}, \mathrm{p}, \mathrm{u}, \mathrm{s},\|\cdot, \cdots, \cdot\|\right) \subset \mathrm{Z}^{2}\left(\mathcal{M}, \Delta_{\mathrm{n}}^{\mathrm{m}}, \mathrm{p}, \mathrm{u}, \mathrm{s},\|\cdot, \cdots, \cdot\|\right)$, for $\mathrm{Z}^{2}=\mathrm{l}_{\infty}^{2}, \mathrm{c}^{2}$ and $\mathrm{c}_{\mathrm{o}}^{2}$.

Proof. We prove the result for the case $Z^{2}=l_{\infty}^{2}$ and the rest of the cases will follow similarly. Let $x=\left(x_{k, l}\right) \in l_{\infty}^{2}\left(\mathcal{M}, \Delta_{n}^{m-1}, p, u, s,\|\cdot, \cdots, \cdot\|\right)$. Then we can have $\rho>0$ such that

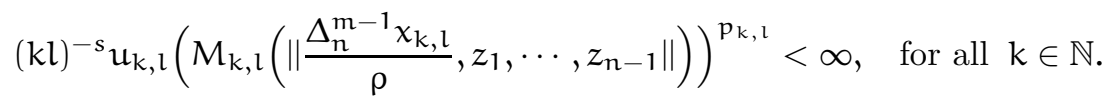

On considering $2 \rho$ and using the convexity of $\left(M_{k, l}\right)$, we have

$$
\begin{aligned}
& (k l)^{-s} u_{k, l} M_{k, l}\left(\left\|\frac{\Delta_{n}^{m} x_{k, l}}{2 \rho}, z_{1}, \cdots, z_{n-1}\right\|\right) \leq \frac{1}{2}(k l)^{-s} u_{k, l} M_{k, l}\left(\left\|\frac{\Delta_{n}^{m-1} x_{k, l}}{\rho}, z_{1}, \cdots, z_{n-1}\right\|\right) \\
& +\frac{1}{2}(k l)^{-s} u_{k, l} M_{k, l}\left(\left\|\frac{\Delta_{n}^{m-1} \chi_{k+n, l+m}}{\rho}, z_{1}, \cdots, z_{n-1}\right\|\right) \text {. }
\end{aligned}
$$

Hence we have

$$
\begin{aligned}
& (k l)^{-s} u_{k, l}\left(M_{k, l}\left(\left\|\frac{\Delta_{n}^{m} x_{k, l}}{2 \rho}, z_{1}, \cdots, z_{n-1}\right\|\right)\right)^{p_{k, l}} \\
& \leq \mathrm{K}\left\{(\mathrm{kl})^{-\mathrm{s}} \mathrm{u}_{\mathrm{k}, \mathrm{l}}\left(\frac{1}{2} M_{k, l}\left(\left\|\frac{\Delta_{\mathrm{n}}^{\mathrm{m}-1} \chi_{k, l}}{\rho}, z_{1}, \cdots, z_{\mathrm{n}-1}\right\|\right)\right)^{\mathrm{p}_{\mathrm{k}, \mathrm{l}}}\right. \\
& \left.+(k l)^{-s} u_{k, l}\left(\frac{1}{2} M_{k, l}\left(\left\|\frac{\Delta_{n}^{m-1} \chi_{k+n, l+m}}{\rho}, z_{1}, \cdots, z_{n-1}\right\|\right)\right)^{p_{k, l}}\right\} \text {. }
\end{aligned}
$$

Then using equation (3.4), we have

$$
(k l)^{-s} u_{k, l}\left(M_{k, l}\left(\left\|\frac{\Delta_{n}^{m} x_{k, l}}{\rho}, z_{1}, \cdots, z_{n-1}\right\|\right)\right)^{p_{k, l}}<\infty, \text { for all } k, l \in \mathbb{N} .
$$

Thus $l_{\infty}^{2}\left(\mathcal{M}, \Delta_{\mathrm{n}}^{\mathrm{m}-1}, p, u, s,\|\cdot, \cdots, \cdot\|\right) \subset l_{\infty}^{2}\left(\mathcal{M}, \Delta_{\mathrm{n}}^{\mathrm{m}}, \mathrm{p}, \mathrm{u}, \mathrm{s},\|\cdot, \cdots, \cdot\|\right)$

Theorem 3.7 Let $\mathcal{M}=\left(M_{k, l}\right)$ be a sequence of Orlicz functions. Then

$$
\mathrm{c}_{0}^{2}\left(\mathcal{M}, \Delta_{\mathrm{n}}^{\mathrm{m}}, \mathrm{p}, \mathrm{u}, \mathrm{s},\|\cdot, \cdots, \cdot\|\right) \subset \mathrm{c}^{2}\left(\mathcal{M}, \Delta_{\mathrm{n}}^{\mathrm{m}}, \mathrm{p}, \mathrm{u}, \mathrm{s},\|\cdot, \cdots, \cdot\|\right) \subset \mathrm{l}_{\infty}^{2}\left(\mathcal{M}, \Delta_{\mathrm{n}}^{\mathrm{m}}, \mathrm{p}, \mathrm{u}, \mathrm{s},\|\cdot, \cdots, \cdot \cdot\|\right) .
$$

Proof. It is obvious that $c_{0}^{2}\left(\mathcal{M}, \Delta_{n}^{m}, p, u, s,\|\cdot, \cdots, \cdot\|\right) \subset c^{2}\left(\mathcal{M}, \Delta_{n}^{m}, p, u, s,\|\cdot, \cdots, \cdot\|\right)$. We shall prove that $c^{2}\left(\mathcal{M}, \Delta_{n}^{m}, p, u, s,\|\cdot, \cdots, \cdot\|\right) \subset l_{\infty}^{2}\left(\mathcal{M}, \Delta_{n}^{m}, p, u, s,\|\cdot, \cdots, \cdot\|\right)$. Let $x=\left(x_{k, l}\right) \in c^{2}\left(\mathcal{M}, \Delta_{\mathfrak{n}}^{m}, p, u, s,\|\cdot, \cdots, \cdot\|\right)$. Then there exists some $\rho>0$ and $L \in X$ such that

$$
\lim _{k, l \rightarrow \infty}(k l)^{-s} u_{k, l}\left(M_{k, l}\left(\left\|\frac{\Delta_{n}^{m} x_{k, l}-L}{\rho}, z_{1}, \cdots, z_{n-1}\right\|\right)\right)^{p_{k, l}}=0 .
$$


On taking $\rho=2 \rho_{1}$, we have

$$
\begin{aligned}
\sup _{k, l}(k l)^{-s} u_{k, l}( & \left.M_{k, l}\left(\| \frac{\Delta_{n}^{m} x_{k, l}}{\rho}, z_{1}, \cdots, z_{n-1}\right)\right)^{p_{k, l}} \\
\leq & \sup _{k, l} K\left[\frac{1}{2}(k l)^{-s} u_{k, l}\left(M_{k, l}\left(\left\|\frac{\Delta_{n}^{m} x_{k, l}-\mathrm{L}}{\rho_{1}}, z_{1}, \cdots, z_{n-1}\right\|\right)\right)\right]^{p_{k, l}} \\
& +\sup _{k, l} K\left[\frac{1}{2}(k l)^{-s} u_{k, l} M_{k, l}\left(\left\|\frac{L}{\rho_{1}}, z_{1}, \cdots, z_{n-1}\right\|\right)\right]^{p_{k, l}} \\
\leq & \sup _{k, l} K\left(\frac{1}{2}\right)^{p_{k, l}}(k l)^{-s} u_{k, l}\left[M_{k, l}\left(\left\|\frac{\Delta_{n}^{m} x_{k, l}-\mathrm{L}}{\rho_{1}}, z_{1}, \cdots, z_{n-1}\right\|\right)\right]^{p_{k, l}} \\
& +\sup _{k, l} K\left(\frac{1}{2}\right)^{p_{k, l}}(k l)^{-s} \max \left(1, u_{k, l}\left(M_{k, l}\left(\left\|\frac{L}{\rho_{1}}, z_{1}, \cdots, z_{n-l}\right\|\right)\right)^{\mathrm{H}}\right),
\end{aligned}
$$

where $H=\max \left(1, \sup p_{k, l}\right)$. Thus we get $x=\left(x_{k, l}\right) \in l_{\infty}^{2}\left(\mathcal{M}, \Delta_{n}^{m}, p, u, s,\|\cdot, \cdots, \cdot\|\right)$. Hence

$$
c_{0}^{2}\left(\mathcal{M}, \Delta_{n}^{m}, p, u, s,\|\cdot, \cdots, \cdot\|\right) \subset c^{2}\left(\mathcal{M}, \Delta_{n}^{m}, p, u, s,\|\cdot, \cdots, \cdot\|\right) \subset l_{\infty}^{2}\left(\mathcal{M}, \Delta_{n}^{m}, p, u, s,\|\cdot, \cdots, \cdot\|\right) .
$$

Theorem 3.8 The sequence space $\mathrm{l}_{\infty}^{2}\left(\mathcal{M}, \Delta_{\mathfrak{n}}^{\mathrm{m}}, \mathrm{p}, \mathrm{u}, \mathrm{s},\|\cdot, \cdots, \cdot\|\right)$ is solid.

Proof. Let $x=\left(x_{k, l}\right) \in l_{\infty}^{2}\left(\mathcal{M}, \Delta_{\mathfrak{n}}^{m}, p, u, s,\|\cdot, \cdots, \cdot\|\right)$, that is

$$
\sup _{k, l \rightarrow \infty}(k l)^{-s} \mathfrak{u}_{k, l}\left[M_{k, l}\left(\left\|\frac{\Delta_{n}^{m} x_{k, l}}{\rho}, z_{1}, \cdots, z_{n-1}\right\|\right)\right]^{p_{k, l}}<\infty .
$$

Let $\left(\alpha_{k, l}\right)$ be a sequence of scalars such that $\left|\alpha_{k, l}\right| \leq 1$ for all $k, l \in \mathbb{N}$. Thus we have

$$
\begin{aligned}
\sup _{k, l \rightarrow \infty}(k l)^{-s} u_{k, l}\left[M_{k, l}\left(\left\|\frac{\alpha_{k, l} \Delta_{\mathfrak{n}}^{m} x_{k, l}}{\rho}, z_{1}, \cdots, z_{\mathfrak{n}-1}\right\|\right)\right]^{p_{k, l}} \\
\quad \leq \sup _{k, l \rightarrow \infty}(k l)^{-s} u_{k, l}\left[M_{k, l}\left(\left\|\frac{\Delta_{n}^{m} x_{k, l}}{\rho}, z_{1}, \cdots, z_{n-1}\right\|\right)\right]^{p_{k, l}}<\infty .
\end{aligned}
$$

This shows that $\left(\alpha_{k, l} x_{k, l}\right) \in l_{\infty}^{2}\left(\mathcal{M}, \Delta_{\mathfrak{n}}^{m}, p, u, s,\|\cdot, \cdots, \cdot\|\right)$ for all sequences of scalars $\left(\alpha_{k, l}\right)$ with $\left|\alpha_{k, l}\right| \leq 1$ for all $k, l \in \mathbb{N}$, whenever $\left(x_{k, l}\right) \in l_{\infty}^{2}\left(\mathcal{M}, \Delta_{\mathfrak{n}}^{m}, p, u, s,\|\cdot, \cdots, \cdot\|\right)$. Hence the space $l_{\infty}^{2}\left(\mathcal{M}, \Delta_{\mathfrak{n}}^{\mathrm{m}}, p, u, s,\|\cdot, \cdots, \cdot\|\right)$ is a solid sequence space.

Theorem 3.9 The sequence space $\mathrm{l}_{\infty}^{2}\left(\mathcal{M}, \Delta_{\mathfrak{n}}^{\mathrm{m}}, \mathrm{p}, \mathrm{u}, \mathrm{s},\|\cdot, \cdots, \cdot\|\right)$ is monotone.

Proof. The proof of the Theorem is obvious and so we omit it.

Received: October 2011. Revised: August 2012. 


\section{References}

[1] B. Altay and F. Başar, Some new spaces of double sequencs, J. Math. Anal. Appl., 309 (2005), $70-90$.

[2] M. Başarir and O. Sonalcan, On some double sequence spaces, J. Indian Acad. Math., 21 (1999), 193-200.

[3] F. Başar and Y. Sever, The space $\mathcal{L}_{\mathrm{p}}$ of double sequences, Math. J. Okayama Univ., 51 (2009), 149-157.

[4] T. J. Bromwich, An introduction to the theory of infinite series, Macmillan and Co. Ltd., New York (1965).

[5] M. Et and R. Çolak, On generalized difference sequence spaces, Soochow J. Math. 21(4) (1995), 377-386.

[6] G. H. Hardy, On the convergence of certain multiple series, Proc. Camb. Phil., Soc., 19 (1917), 86-95.

[7] G. H. Hardy, Divergent series, Oxford at the Clarendon Press, (1949).

[8] S. Gähler, Linear 2-normietre Rume, Math. Nachr., 28 (1965), 1-43.

[9] H. Gunawan, On n-inner product, n-norms and the Cauchy-Schwartz inequality, Sci. Math. Jap., 5 (2001), 47-54.

[10] H. Gunawan, The space of $\mathrm{p}$-summable sequence and its natural n-norm, Bull. Aust. Math. Soc., 64 (2001), 137-147.

[11] H. Gunawan and M. Mashadi, On n-normed spaces, Int. J. Math. Math. Sci., 27 (2001), 631639.

[12] P. K. Kamthan and M. Gupta, Sequence spaces and series, Lecture Notes in Pure and Applied Mathematics, 65 Marcel Dekker, Inc., New York,(1981).

[13] H. Kızmaz, On certain sequence spaces, Canad. Math-Bull., 24 (1981), 169-176.

[14] J. Lindenstrauss and L. Tzafriri, On Orlicz seequence spaces, Israel J. Math, 10 (1971), 379390.

[15] L. Maligranda, Orlicz spaces and interpolation, Seminars in Mathematics 5, Polish Academy of Science, (1989).

[16] A. Misiak, n-inner product spaces, Math. Nachr., 140 (1989), 299-319.

[17] F. Moricz, Extension of the spaces $\mathrm{c}$ and $\mathrm{c}_{\mathrm{o}}$ from single to double sequences, Acta Math. Hungarica, 57 (1991), 129-136. 
[18] F. Moricz and B. E. Rhoades, Almost convergence of double sequences and strong reqularity of summability matrices, Math. Proc. Camb. Phil. Soc., 104 (1988), 283-294.

[19] M. Mursaleen, Almost strongly regular matrices and a core theorem for double sequences, J. Math. Anal. Appl., 293(2) (2004), 523-531.

[20] M. Mursaleen, M. A. Khan and Qamaruddin, Difference sequence spaces defined by Orlicz functions, Demonstratio Math., XXXII (1999), 145-150.

[21] M. Mursaleen and O. H. H. Edely, Statistical convergence of double sequences, J. Math. Anal. Appl., 288(1) (2003), 223-231.

[22] M. Mursaleen and O. H. H. Edely, Almost convergence and a core theorem for double sequences, J. Math. Anal. Appl., 293(2) (2004), 532-540.

[23] J. Musielak, Orlicz spaces and modular spaces, Lecture Notes in Mathematics, 1034 (1983).

[24] H. Nakano, Modular sequence spaces, Proc. Japan Acad., 27 (1951), 508-512.

[25] S. D. Prashar and B. Choudhary, Sequence spaces defined by Orlicz functions, Indiana J. Pure Appl. Math. 25(14) (1994), 419-428.

[26] A. Pringsheim, Zur Theori der zweifach unendlichen Zahlenfolgen, Math. Ann. 53(1900), 289-321.

[27] K. Raj, A. K. Sharma and S. K. Sharma, A Sequence space defined by a Musielak-Orlicz function, Int. J. Pure Appl. Math., 67 (2011), 475-484 .

[28] K. Raj, S. K. Sharma and A. K. Sharma, Difference sequence spaces in $\mathrm{n}$-normed spaces defined by a Musielak-Orlicz function, Armen. j. Math., 3 (2010), 127-141.

[29] K. Raj and S. K. Sharma, Some sequence spaces in 2-normed spaces defined by a MusielakOrlicz function, Acta Univ. Sapientiae Math. 3 (2011), 97-109.

[30] G. M. Robinson, Divergent double sequences and series, Trans. Amer. Math. Soc. 28(1926), 50-73.

[31] L. L. Silverman, On the definition of the sum of a divergent series, Ph. D. Thesis, University of Missouri Studies, Mathematics Series, (1913).

[32] S. Simons, The sequence spaces $l\left(\mathrm{p}_{v}\right)$ and $\mathfrak{m}\left(\mathrm{p}_{v}\right)$, Proc. Japan Acad., 27 (1951), 508-512.

[33] B. C. Tripathy, Generalized difference paranormed statistically convergent sequences defined by Orlicz function in a locally convex spaces, Soochow J. Math., 30 (2004), 431-446.

[34] B. C. Tripathy, Statistically convergent double sequences, Tamkang J. Math., 34 (2003), 231-237. 
[35] A. Wilansky, Summability through Functional Analysis, North- Holland Math. Stud. 85(1984).

[36] M. Zeltser, Investigation of double sequence spaces by Soft and Hard Analytical Methods, Dissertationes Mathematicae Universitatis Tartuensis 25, Tartu University Press, Univ. of Tartu, Faculty of Mathematics and Computer Science, Tartu (2001). 